\title{
Comparative analysis among standards of the area calculation of transversal reinforcement on reinforced concrete beams of high resistance subjected by shear force
}

\section{Análise comparativa entre normas do cálculo da área da armadura transversal em vigas de concreto armado de resistência elevada submetidas à ação de força cortante}

D. H. L. BRAZ a

danielhlbraz@gmail.com https://orcid.org/0000-0003-2545-6058

R. BARROS b

rodrigobarros@ect.ufrn.br https://orcid.org/0000-0002-7218-2646

J. N. DA SILVA FILHO a,c jneres@ect.ufrn.br https://orcid.org/0000-0002-9138-1771

\begin{abstract}
High strength concretes (HSC) correspond to a characteristic compression strength between 55 e $90 \mathrm{MPa}$. With the growing use of HSC, studies about the regular design standards of elements made of it, specifically standards about design on shear, become necessary. Hence, the main aspects of the NBR, Model Code 1990 e 2010, Portuguese Standard and German Standard related to the design on shear are presented. From the numerical simulations, with the addition of Cladera and Mari's experimental contributions, it is confirmed that the Brazilian design standard procedure produces lower transverse reinforcement areas in comparison to the ones predicted by the international codes; these, excepted by LoA III, do not consider the concrete contribution, in spite of being experimentally verified, leading to very conservative results.
\end{abstract}

Keywords: design, shear, high strength.

\section{Resumo}

Concretos de alta resistência (CAR) correspondem a uma resistência à compressão característica compreendida entre 55 e $90 \mathrm{MPa}$. Com a possibilidade crescente da utilização de CAR, faz-se necessária a realização de estudos que abordem os tratamentos normativos usuais acerca do dimensionamento de elementos por ele constituídos, especificamente, à ação de força cortante. Portanto, são apresentadas os principais aspectos da NBR, Model Code 1990 e 2010, Norma portuguesa e alemã acerca dos dimensionamento à cortante. Das simulações numéricas, acrescidas das contribuições experimentais de Cladera e Marí, constata-se que o procedimento de cálculo da NBR produz áreas de estribos inferiores às previstas pelos códigos internacionais; estes, com exceção do LoA III, não adotam a contribuição do concreto, apesar de esta ser verificada experimentalmente, levando a resultados muito conservadores.

Palavras-chave: dimensionamento, cortante, alta resistência. 


\section{Introduction}

High strength concretes (HSC) correspond to a characteristic compression strength $\left(f_{\mathrm{ck}}\right)$ between 55 e $90 \mathrm{MPa}$, according to ABNT NBR 6118:2014 [5]. Its use has been disseminated due to the demand for structures for which weight reduction is important and/or for when the architecture imposes the use of more slender pieces (Silva [1]). HSC are obtained by improving the compaction of the concrete mixtures, which improves the resistance of the paste and its interface with coarse aggregates (Cladera [2]).

From the analysis of the standard's procedures, the importance of the concrete class for the design of the elements is prominent. Thus, with the increased possibility of using concretes from group II, it is necessary to conduct studies that approach the usual codes' treatments regarding the design of high strength concrete elements, specifically the design on shear. According to Arslan [3], the
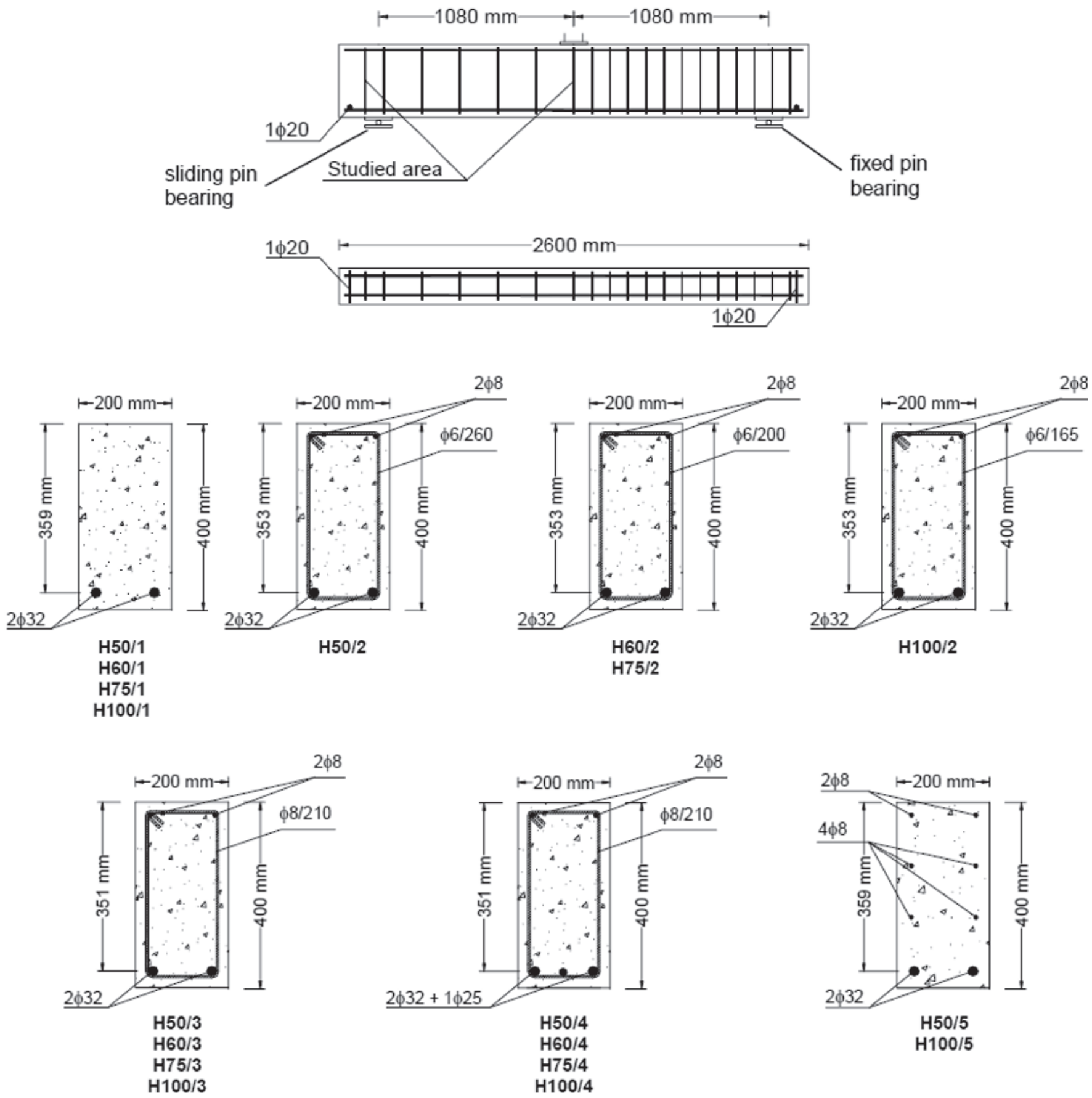

Figure 1

Test set-up and cross-section of the beam specimens. (Cladera [2]) 


\section{Table 1}

ANN for beams with web reinforcement. Ranges of parameters in the database [4]

\begin{tabular}{ccc}
\hline Parameter & Minimum & Maximum \\
\hline$d(\mathrm{~mm})$ & 198 & 925 \\
$\mathrm{~d} / \mathrm{b}$ & 0.792 & 4.5 \\
$\rho_{\mathrm{l}}(\%)$ & 0.50 & 5.80 \\
$\rho_{\mathrm{w}}(\mathrm{MPa})$ & 0.33 & 3.57 \\
$\mathrm{f}_{\mathrm{c}}(\mathrm{MPa})$ & 21 & 125.2 \\
$\mathrm{~d} / \mathrm{d}$ & 2.49 & 5.00 \\
$V(\mathrm{kN})$ & 63.28 & 1172.19 \\
\hline
\end{tabular}

concrete's contribution is important in the design of beams where the factored shear force is close to the value of the shear force required to produce diagonal tension cracking, and also necessary for the economic design of beams and slabs with little or no shear reinforcement.

Among the experimental studies regarding the high strength concrete beams subjected to shear force, those of Cladera [2] and Cladera \& Mari [4] are highlighted. In the first, 18 beams of reinforced concrete beams - which characteristics are illustrated in Figure 1 - with compressive strength between 50 and $87 \mathrm{MPa}$, were tested at the Structural Technology Laboratory of the Department of Construction Engineering at the School of Civil Engineering of Barcelona. The main objectives of the experimental program were to study the influence of concrete compressive strength in beams with and without shear reinforcement; to propose and verify a more adequate minimum amount of web reinforcement then the proposed by the Spanish code EHE Instrución de Hormigón Estructural of 1998; to evaluate the efficiency of the amount of shear and longitudinal reinforcement as a function of $f_{c k}$; and to study the influence of the longitudinally-distributed web reinforcement in beams without stirrups.

In the second [4], Eurocode 2, AASHTO LRFD and ACI 318-02 were evaluated with an Artificial Neural Network (ANN) based on 123 test-beams of high strength concrete. From the results of the ANN, the authors analyzed the influences of the amount of transverse web reinforcement, the effect of the beams' size and effective depth, of the concrete compressive strength, of the amount of longitudinal reinforcement and the ratio between shear span and the effective depth on the shear strength. Hence, an alternate design method was proposed. The ANN contemplated the testbeams with the characteristics indicated in Table 1.

\subsection{Justification}

The expansion of the use of high strength concretes indicates the need for better understanding the structural behavior of the elements made of it. This understanding comprehends the usual design standard's procedures. From the numerical analyses and simulations, comparisons are made to explain how each code approaches the issue, specifically about shear design. In addition, the experimental contributions of Cladera [2] and Cladera and Marí [4] will base the comparative analyses between the standard's predictions of shear reinforcement area and those demanded according to experimental results. The ultimate shear forces, the experi- mentally predicted and the obtained through normative calculation, are also contemplated in the analysis.

\section{Analysed design standards}

\subsection{ABNT NBR 6118:2014 [5]}

The Brazilian code presents two design models for linear elements subjected to shear force. For both, the minimum ratio of web reinforcement is given by:

$\rho_{\mathrm{sw}}=\frac{\mathrm{A}_{\mathrm{sw}}}{b_{\mathrm{w}} \cdot s \cdot \operatorname{sen} \alpha} \geq 0,2 \frac{f_{\mathrm{ct}, \mathrm{m}}}{f_{\mathrm{ywk}}}$

where:

$A_{s w}$ is the shear reinforcement area;

$s$ is the longitudinal space between stirrups, along the longitudinal axis of the structural element;

$\alpha$ is the inclination of the transverse reinforcement related to the longitudinal axis of the structural element; it is contained in the interval $45^{\circ} \leq \alpha \leq 90^{\circ}$;

$b_{\mathrm{w}}$ is the effective web width;

$f_{\text {ywk }}$ is the characteristic value of yield strength of the reinforcing steel; $f_{\mathrm{ct}, \mathrm{m}}=2,12 \ln \left(1+0,11 f_{\mathrm{ck}}\right)$ for concretes C55 até C90.

The resistance is considered satisfactory when simultaneously observes the following conditions:

$V_{\mathrm{Sd}} \leq V_{\mathrm{Rd} 2}$

$V_{\mathrm{Sd}} \leq V_{\mathrm{Rd} 3}=V_{\mathrm{c}}+V_{\mathrm{sw}}$

where:

$V_{S d}$ is the design value of applied shear force;

$V_{\mathrm{Rd} 2}$ is the design shear strength related to the concrete failure by diagonal compression;

$V_{\mathrm{Rd} 3}$ is the design shear force related to the diagonal tension failure, where $V_{c}$ is the amount of shear force absorbed by the complementary mechanisms of the truss and $V_{\mathrm{sw}}$ the amount of shear force resisted by the transverse reinforcement.

\subsubsection{Calculation Model I}

This Model, conducted by the following expressions, admits struts' inclination of $\theta=45^{\circ}$ in relation to the longitudinal axis of the structural element and complementary portion $V_{c}$ constant and independent from $V_{S d}$

a) Verification of the concrete failure by diagonal compression:

$V_{\mathrm{Rd} 2}=0,27 \propto_{\mathrm{v} 2} f_{\mathrm{cd}} b_{\mathrm{w}} d$

where:

$\propto_{v 2}=\left(1-f_{c k} / 250\right)$ and $f_{c k}$ in MPa;

b) Calculation of the transverse reinforcement:

$V_{\mathrm{Rd} 3}=V_{\mathrm{c}}+V_{\mathrm{sw}}$

where:

$V_{s w}=\left(A_{s w} / s\right) 0,9 d f_{y w d}(\operatorname{sen} \alpha+\cos \alpha)$

$\mathrm{V}_{\mathrm{c}}=\mathrm{V}_{\mathrm{c} 0}=0,6 \mathrm{f}_{\mathrm{ctd}} \mathrm{b}_{\mathrm{w}} \mathrm{d}$

$\mathrm{f}_{\mathrm{ctd}}=\mathrm{f}_{\mathrm{ctk}, \mathrm{inf}} / \gamma_{\mathrm{c}}=0,7 \mathrm{f}_{\mathrm{ct}, \mathrm{m}} / \gamma_{\mathrm{c}}$ 
where:

$d$ is the distance between the compressed edge and the center of gravity of the reinforcement;

$f_{\text {ywd }}$ is the stress on the passive transverse reinforcement not superior to $435 \mathrm{MPa}$.

\subsubsection{Calculation Model II}

This Model admits $\theta$ inclinations between $30^{\circ}$ and $45^{\circ}$ and the decrease of $V_{c}$ with the increase of $V_{\text {Sd }}$ a) Verification of the concrete failure by diagonal compression:

$V_{\mathrm{Rd} 2}=0,54 \propto_{\mathrm{v} 2} f_{\mathrm{cd}} b_{\mathrm{w}} d \operatorname{sen}^{2} \theta(\operatorname{cotg} \alpha+\operatorname{cotg} \theta)$

b) Calculation of the transverse reinforcement, as shown on Equation 5, where:

$\mathrm{V}_{\mathrm{sw}}=\left(\mathrm{A}_{\mathrm{sw}} / \mathrm{s}\right) 0,9 \mathrm{df}_{\mathrm{ywd}}(\operatorname{cotg} \alpha+\operatorname{cotg} \theta) \operatorname{sen} \alpha$;

$\mathrm{V}_{\mathrm{c} 1}=\mathrm{V}_{\mathrm{c} 0}$ when $\mathrm{V}_{\mathrm{Sd}} \leq \mathrm{V}_{\mathrm{c} 0}$;

$\mathrm{V}_{\mathrm{c} 1}=0$ when $\mathrm{V}_{\mathrm{Sd}}=\mathrm{V}_{\mathrm{Rd} 2}$;

$V_{\mathrm{c} 1}=\left(\frac{V_{\mathrm{Rd} 2}-V_{\mathrm{Sd}}}{V_{\mathrm{Rd} 2}-V_{\mathrm{c} 0}}\right) V_{\mathrm{c} 0}$ for intermediate values.

Table 2

Results of Model I of ABNT NBR 61 18:2014

\begin{tabular}{|c|c|c|c|c|c|c|c|c|c|}
\hline $\begin{array}{c}f_{c k} \\
(M P a)\end{array}$ & $\begin{array}{c}f_{\text {ctimm }} \\
(\mathrm{MPa})\end{array}$ & $\begin{array}{c}f_{\text {ctd }} \\
(\mathrm{MPa})\end{array}$ & $\begin{array}{c}b_{w} \\
(\mathrm{~cm})\end{array}$ & $\begin{array}{c}d \\
(\mathrm{~cm})\end{array}$ & $\begin{array}{l}V_{\text {sd }} \\
(k N)\end{array}$ & $\begin{array}{l}V_{\mathrm{Rd} 2} \\
(\mathrm{kN})\end{array}$ & $\begin{array}{l}V_{c 0} \\
(k N)\end{array}$ & $\begin{array}{l}V_{\text {sw }} \\
(k N)\end{array}$ & $\begin{array}{c}A_{\mathrm{sw}} \\
\left(\mathrm{cm}^{2} / \mathrm{m}\right)\end{array}$ \\
\hline 55 & 4.14 & 2.07 & 12 & 40 & 100.00 & 397.13 & 59.62 & 40.38 & 2.58 \\
\hline 55 & 4.14 & 2.07 & 12 & 40 & 125.00 & 397.13 & 59.62 & 65.38 & 4.17 \\
\hline 55 & 4.14 & 2.07 & 12 & 40 & 150.00 & 397.13 & 59.62 & 90.38 & 5.77 \\
\hline 55 & 4.14 & 2.07 & 12 & 40 & 175.00 & 397.13 & 59.62 & 115.38 & 7.37 \\
\hline 60 & 4.30 & 2.15 & 12 & 40 & 100.00 & 422.13 & 61.92 & 38.08 & 2.43 \\
\hline 60 & 4.30 & 2.15 & 12 & 40 & 125.00 & 422.13 & 61.92 & 63.08 & 4.03 \\
\hline 60 & 4.30 & 2.15 & 12 & 40 & 150.00 & 422.13 & 61.92 & 88.08 & 5.62 \\
\hline 60 & 4.30 & 2.15 & 12 & 40 & 175.00 & 422.13 & 61.92 & 113.08 & 7.22 \\
\hline 70 & 4.59 & 2.29 & 12 & 40 & 100.00 & 466.56 & 66.04 & 33.96 & 2.20 \\
\hline 70 & 4.59 & 2.29 & 12 & 40 & 125.00 & 466.56 & 66.04 & 58.96 & 3.76 \\
\hline 70 & 4.59 & 2.29 & 12 & 40 & 150.00 & 466.56 & 66.04 & 83.96 & 5.36 \\
\hline 70 & 4.59 & 2.29 & 12 & 40 & 175.00 & 466.56 & 66.04 & 108.96 & 6.96 \\
\hline 80 & 4.84 & 2.42 & 12 & 40 & 100.00 & 503.59 & 69.68 & 30.32 & 2.32 \\
\hline 80 & 4.84 & 2.42 & 12 & 40 & 125.00 & 503.59 & 69.68 & 55.32 & 3.53 \\
\hline 80 & 4.84 & 2.42 & 12 & 40 & 150.00 & 503.59 & 69.68 & 80.32 & 5.13 \\
\hline 80 & 4.84 & 2.42 & 12 & 40 & 175.00 & 503.59 & 69.68 & 105.32 & 6.73 \\
\hline 90 & 5.06 & 2.53 & 12 & 40 & 100.00 & 533.21 & 72.92 & 27.08 & 2.43 \\
\hline 90 & 5.06 & 2.53 & 12 & 40 & 125.00 & 533.21 & 72.92 & 52.08 & 3.33 \\
\hline 90 & 5.06 & 2.53 & 12 & 40 & 150.00 & 533.21 & 72.92 & 77.08 & 4.92 \\
\hline 90 & 5.06 & 2.53 & 12 & 40 & 175.00 & 533.21 & 72.92 & 102.08 & 6.52 \\
\hline
\end{tabular}

\section{Table 3}

Results of Model II of ABNT NBR 6118:2014

\begin{tabular}{|c|c|c|c|c|c|c|c|c|c|c|}
\hline $\begin{array}{c}f_{c k} \\
(M P a)\end{array}$ & $\begin{array}{c}f_{c t, m} \\
\text { (MPa) }\end{array}$ & $\begin{array}{c}f_{\text {ctd }} \\
(\mathrm{MPa})\end{array}$ & $\theta^{\circ}$ & $\begin{array}{c}b_{w} \\
(\mathrm{~cm})\end{array}$ & $\begin{array}{c}d \\
(\mathrm{~cm})\end{array}$ & $\begin{array}{c}V_{s d} \\
(k N)\end{array}$ & $\begin{array}{l}\mathrm{V}_{\mathrm{Rd} 2} \\
(\mathrm{kN}) \\
\end{array}$ & $\begin{array}{l}\mathrm{V}_{\mathrm{cl}} \\
(\mathrm{kN})\end{array}$ & $\begin{array}{l}\mathrm{V}_{\mathrm{sw}} \\
(\mathrm{kN})\end{array}$ & $\begin{array}{c}A_{\mathrm{sw}} \\
\left(\mathrm{cm}^{2} / \mathrm{m}\right) \\
\end{array}$ \\
\hline 55 & 4.14 & 2.07 & 45 & 12 & 40 & 100.00 & 397.13 & 52.49 & 47.51 & 3.03 \\
\hline 55 & 4.14 & 2.07 & 45 & 12 & 40 & 125.00 & 397.13 & 48.07 & 76.93 & 4.91 \\
\hline 55 & 4.14 & 2.07 & 45 & 12 & 40 & 150.00 & 397.13 & 43.66 & 106.34 & 6.79 \\
\hline 55 & 4.14 & 2.07 & 45 & 12 & 40 & 175.00 & 397.13 & 39.24 & 135.76 & 8.67 \\
\hline 60 & 4.30 & 2.15 & 45 & 12 & 40 & 100.00 & 422.13 & 55.37 & 44.63 & 2.85 \\
\hline 60 & 4.30 & 2.15 & 45 & 12 & 40 & 125.00 & 422.13 & 51.07 & 73.93 & 4.72 \\
\hline 60 & 4.30 & 2.15 & 45 & 12 & 40 & 150.00 & 422.13 & 46.77 & 103.23 & 6.59 \\
\hline 60 & 4.30 & 2.15 & 45 & 12 & 40 & 175.00 & 422.13 & 42.48 & 132.52 & 8.46 \\
\hline 70 & 4.59 & 2.29 & 45 & 12 & 40 & 100.00 & 466.56 & 60.44 & 39.56 & 2.53 \\
\hline 70 & 4.59 & 2.29 & 45 & 12 & 40 & 125.00 & 466.56 & 56.32 & 68.68 & 4.39 \\
\hline 70 & 4.59 & 2.29 & 45 & 12 & 40 & 150.00 & 466.56 & 52.20 & 97.80 & 6.25 \\
\hline 70 & 4.59 & 2.29 & 45 & 12 & 40 & 175.00 & 466.56 & 48.08 & 126.92 & 8.11 \\
\hline 80 & 4.84 & 2.42 & 45 & 12 & 40 & 100.00 & 503.59 & 64.81 & 35.19 & 2.32 \\
\hline 80 & 4.84 & 2.42 & 45 & 12 & 40 & 125.00 & 503.59 & 60.79 & 64.21 & 4.10 \\
\hline 80 & 4.84 & 2.42 & 45 & 12 & 40 & 150.00 & 503.59 & 56.78 & 93.22 & 5.95 \\
\hline 80 & 4.84 & 2.42 & 45 & 12 & 40 & 175.00 & 503.59 & 52.76 & 122.24 & 7.81 \\
\hline 90 & 5.06 & 2.53 & 45 & 12 & 40 & 100.00 & 533.21 & 68.63 & 31.37 & 2.43 \\
\hline 90 & 5.06 & 2.53 & 45 & 12 & 40 & 125.00 & 533.21 & 64.67 & 60.33 & 3.85 \\
\hline 90 & 5.06 & 2.53 & 45 & 12 & 40 & 150.00 & 533.21 & 60.71 & 89.29 & 5.70 \\
\hline 90 & 5.06 & 2.53 & 45 & 12 & 40 & 175.00 & 533.21 & 56.75 & 118.25 & 7.55 \\
\hline
\end{tabular}




\subsection{CEB-FIP Model Code 1990 [6]}

The model code of 1990 brings in its section 6.3.3 Shear and axial action effects the considerations presented below. This standard establishes the $\theta$ inclination of the struts between $18.4^{\circ}$ and $45^{\circ}$.

a) Minimum transverse reinforcement ratio:

$\omega_{\mathrm{sw}}=\frac{A_{\mathrm{sw}} \cdot f_{\mathrm{yk}}}{b_{\mathrm{w}} \cdot s \cdot f_{\mathrm{ctm}} \cdot \operatorname{sen} \alpha} \geq 0,2$ where:

$$
\begin{aligned}
& f_{\text {ctm }}=f_{c t k o, m}\left(\frac{f_{c k}}{f_{c k o}}\right)^{2 / 3} \\
& \mathrm{f}_{\text {cko }}=10 \mathrm{MPa} \\
& \mathrm{f}_{\text {ctko,m }}=1,40 \mathrm{MPa}
\end{aligned}
$$

b) Maximum shear resistance, with $\theta=45^{\circ}$ :

$V_{\mathrm{Rd}, \text { max }}=\frac{f_{\mathrm{cd} 2}}{2} b_{\mathrm{w}} z(1+\operatorname{cotg} \propto)$

where:

$$
f_{\mathrm{cd} 2}=0,60\left[1-\frac{f_{c k}}{250}\right] f_{c d}
$$

\begin{tabular}{|c|c|c|c|c|c|c|c|c|c|}
\hline $\begin{array}{c}f_{c k} \\
(M P a)\end{array}$ & $\begin{array}{c}\mathrm{f}_{\mathrm{ct}, \mathrm{m}} \\
(\mathrm{MPa})\end{array}$ & $\begin{array}{c}f_{c d 2} \\
(M P a)\end{array}$ & $\begin{array}{c}b_{w} \\
(\mathrm{~cm})\end{array}$ & $\begin{array}{c}d \\
(\mathrm{~cm})\end{array}$ & $\theta^{\circ}$ & $\begin{array}{l}\mathrm{V}_{\mathrm{Rd}, \max } \\
(\mathrm{kNN})\end{array}$ & $\begin{array}{c}V_{s d} \\
(k N)\end{array}$ & $\begin{array}{c}F_{\text {Stw }} \\
(\mathrm{kN})\end{array}$ & $\begin{array}{c}A_{\mathrm{sw}} \\
\left(\mathrm{cm}^{2} / \mathrm{m}\right)\end{array}$ \\
\hline 55 & 4.36 & 17.16 & 12 & 40 & 45 & 370.66 & 100.00 & 100.00 & 6.39 \\
\hline 55 & 4.36 & 17.16 & 12 & 40 & 45 & 370.66 & 125.00 & 125.00 & 7.99 \\
\hline 55 & 4.36 & 17.16 & 12 & 40 & 45 & 370.66 & 150.00 & 150.00 & 9.58 \\
\hline 55 & 4.36 & 17.16 & 12 & 40 & 45 & 370.66 & 175.00 & 175.00 & 11.18 \\
\hline 60 & 4.62 & 18.24 & 12 & 40 & 45 & 393.98 & 100.00 & 100.00 & 6.39 \\
\hline 60 & 4.62 & 18.24 & 12 & 40 & 45 & 393.98 & 125.00 & 125.00 & 7.99 \\
\hline 60 & 4.62 & 18.24 & 12 & 40 & 45 & 393.98 & 150.00 & 150.00 & 9.58 \\
\hline 60 & 4.62 & 18.24 & 12 & 40 & 45 & 393.98 & 175.00 & 175.00 & 11.18 \\
\hline 70 & 5.12 & 20.16 & 12 & 40 & 45 & 435.46 & 100.00 & 100.00 & 6.39 \\
\hline 70 & 5.12 & 20.16 & 12 & 40 & 45 & 435.46 & 125.00 & 125.00 & 7.99 \\
\hline 70 & 5.12 & 20.16 & 12 & 40 & 45 & 435.46 & 150.00 & 150.00 & 9.58 \\
\hline 70 & 5.12 & 20.16 & 12 & 40 & 45 & 435.46 & 175.00 & 175.00 & 11.18 \\
\hline 80 & 5.60 & 21.76 & 12 & 40 & 45 & 470.02 & 100.00 & 100.00 & 6.39 \\
\hline 80 & 5.60 & 21.76 & 12 & 40 & 45 & 470.02 & 125.00 & 125.00 & 7.99 \\
\hline 80 & 5.60 & 21.76 & 12 & 40 & 45 & 470.02 & 150.00 & 150.00 & 9.58 \\
\hline 80 & 5.60 & 21.76 & 12 & 40 & 45 & 470.02 & 175.00 & 175.00 & 11.18 \\
\hline 90 & 6.06 & 23.04 & 12 & 40 & 45 & 497.66 & 100.00 & 100.00 & 6.39 \\
\hline 90 & 6.06 & 23.04 & 12 & 40 & 45 & 497.66 & 125.00 & 125.00 & 7.99 \\
\hline 90 & 6.06 & 23.04 & 12 & 40 & 45 & 497.66 & 150.00 & 150.00 & 9.58 \\
\hline 90 & 6.06 & 23.04 & 12 & 40 & 45 & 497.66 & 175.00 & 175.00 & 11.18 \\
\hline
\end{tabular}

\section{Table 4}

Results of MC 1990

\begin{tabular}{|c|c|c|c|c|c|c|c|c|c|c|}
\hline $\begin{array}{c}f_{c k} \\
(M P a)\end{array}$ & $\begin{array}{c}\mathrm{f}_{\mathrm{ct}, \mathrm{m}} \\
(\mathrm{MPa})\end{array}$ & $\begin{array}{c}b_{w} \\
(\mathrm{~cm})\end{array}$ & $\begin{array}{c}d \\
(\mathrm{~cm})\end{array}$ & $\theta^{\circ}$ & $\mathbf{k}_{\varepsilon}$ & $\mathrm{k}_{\mathrm{c}}$ & $\begin{array}{l}\mathrm{V}_{\mathrm{Ed}} \\
(\mathrm{kN})\end{array}$ & $\begin{array}{l}\mathrm{V}_{\mathrm{Rd}, \max } \\
(\mathrm{kN})\end{array}$ & $\begin{array}{l}V_{\text {Rd,s }} \\
(\mathrm{kN})\end{array}$ & $\begin{array}{c}A_{\mathrm{sw}} \\
\left(\mathrm{cm}^{2} / \mathrm{m}\right)\end{array}$ \\
\hline 55 & 4.21 & 12 & 40 & 45 & \multirow{4}{*}{0.55} & \multirow{4}{*}{0.45} & 100.00 & 355.91 & 100.00 & 6.39 \\
\hline 55 & 4.21 & 12 & 40 & 45 & & & 125.00 & 355.91 & 125.00 & 7.99 \\
\hline 55 & 4.21 & 12 & 40 & 45 & & & 150.00 & 355.91 & 150.00 & 9.58 \\
\hline 55 & 4.21 & 12 & 40 & 45 & & & 175.00 & 355.91 & 175.00 & 11.18 \\
\hline 60 & 4.35 & 12 & 40 & 45 & \multirow{4}{*}{0.55} & \multirow{4}{*}{0.44} & 100.00 & 377.17 & 100.00 & 6.39 \\
\hline 60 & 4.35 & 12 & 40 & 45 & & & 125.00 & 377.17 & 125.00 & 7.99 \\
\hline 60 & 4.35 & 12 & 40 & 45 & & & 150.00 & 377.17 & 150.00 & 9.58 \\
\hline 60 & 4.35 & 12 & 40 & 45 & & & 175.00 & 377.17 & 175.00 & 11.18 \\
\hline 70 & 4.61 & 12 & 40 & 45 & \multirow{4}{*}{0.55} & \multirow{4}{*}{0.41} & 100.00 & 417.99 & 100.00 & 6.39 \\
\hline 70 & 4.61 & 12 & 40 & 45 & & & 125.00 & 417.99 & 125.00 & 7.99 \\
\hline 70 & 4.61 & 12 & 40 & 45 & & & 150.00 & 417.99 & 150.00 & 9.58 \\
\hline 70 & 4.61 & 12 & 40 & 45 & & & 175.00 & 417.99 & 175.00 & 11.18 \\
\hline 80 & 4.84 & 12 & 40 & 45 & \multirow{4}{*}{0.55} & \multirow{4}{*}{0.40} & 100.00 & 456.90 & 100.00 & 6.39 \\
\hline 80 & 4.84 & 12 & 40 & 45 & & & 125.00 & 456.90 & 125.00 & 7.99 \\
\hline 80 & 4.84 & 12 & 40 & 45 & & & 150.00 & 456.90 & 150.00 & 9.58 \\
\hline 80 & 4.84 & 12 & 40 & 45 & & & 175.00 & 456.90 & 175.00 & 11.18 \\
\hline 90 & 5.04 & 12 & 40 & 45 & \multirow{4}{*}{0.55} & \multirow{4}{*}{0.38} & 100.00 & 494.23 & 100.00 & 6.39 \\
\hline 90 & 5.04 & 12 & 40 & 45 & & & 125.00 & 494.23 & 125.00 & 7.99 \\
\hline 90 & 5.04 & 12 & 40 & 45 & & & 150.00 & 494.23 & 150.00 & 9.58 \\
\hline 90 & 5.04 & 12 & 40 & 45 & & & 175.00 & 494.23 & 175.00 & 11.18 \\
\hline
\end{tabular}

Table 5

Results of MC 2010 for LoA I 
c) Tension of web steel:

$F_{S t w}=\frac{V_{S d}}{\operatorname{sen} \propto}$

$F_{R t w}=\left[\frac{A_{s w} \cdot f_{y d}}{\mathrm{~s}}\right] z(\operatorname{cotg} \theta+\operatorname{cotg} \alpha)$

where:

$F_{S t w}$ is the applied shear force on the transverse reinforcement;

$F_{R t w}$ is the resistant shear force of the reinforcement.

\subsection{CEB-FIP Model Code 2010 [7]}

Ahead, the considerations of the 2010 version of the model code are presented.

a) Minimum shear reinforcement area:

$A_{\mathrm{sw}, \min }=0,08 \cdot \sqrt{f_{c k}} \cdot \frac{b_{w} \cdot s_{w}}{f_{y k}}$

b) Design shear resistance:

$V_{R d}=V_{R d, c}+V_{R d, s} \geq V_{E d}$

Table 6

Results of MC 2010 for LoA II

\begin{tabular}{|c|c|c|c|c|c|c|c|c|}
\hline $\begin{array}{c}f_{c k} \\
(M P a)\end{array}$ & $\begin{array}{c}b_{w} \\
(\mathrm{~cm})\end{array}$ & $\begin{array}{c}d \\
(\mathrm{~cm})\end{array}$ & $\mathbf{k}_{\varepsilon}$ & $\mathrm{k}_{\mathrm{c}}$ & $\begin{array}{c}V_{E d} \\
(k N)\end{array}$ & $\begin{array}{l}\mathrm{V}_{\mathrm{Rd}, \max } \\
(\mathrm{kN})\end{array}$ & $\begin{array}{l}V_{\text {Rd,s }} \\
(\mathrm{kN})\end{array}$ & $\begin{array}{c}A_{\mathrm{sw}} \\
\left(\mathrm{cm}^{2} / \mathrm{m}\right)\end{array}$ \\
\hline 55 & 12 & 40 & \multirow{4}{*}{0.65} & \multirow{4}{*}{0.53} & 100.00 & 420.62 & 100.00 & 6.39 \\
\hline 55 & 12 & 40 & & & 125.00 & 420.62 & 125.00 & 7.99 \\
\hline 55 & 12 & 40 & & & 150.00 & 420.62 & 150.00 & 9.58 \\
\hline 55 & 12 & 40 & & & 175.00 & 420.62 & 175.00 & 11.18 \\
\hline 60 & 12 & 40 & \multirow{4}{*}{0.65} & \multirow{4}{*}{0.52} & 100.00 & 445.74 & 100.00 & 6.39 \\
\hline 60 & 12 & 40 & & & 125.00 & 445.74 & 125.00 & 7.99 \\
\hline 60 & 12 & 40 & & & 150.00 & 445.74 & 150.00 & 9.58 \\
\hline 60 & 12 & 40 & & & 175.00 & 445.74 & 175.00 & 11.18 \\
\hline 70 & 12 & 40 & \multirow{4}{*}{0.65} & \multirow{4}{*}{0.49} & 100.00 & 493.99 & 100.00 & 6.39 \\
\hline 70 & 12 & 40 & & & 125.00 & 493.99 & 125.00 & 7.99 \\
\hline 70 & 12 & 40 & & & 150.00 & 493.99 & 150.00 & 9.58 \\
\hline 70 & 12 & 40 & & & 175.00 & 493.99 & 175.00 & 11.18 \\
\hline 80 & 12 & 40 & \multirow{4}{*}{0.65} & \multirow{4}{*}{0.47} & 100.00 & 539.98 & 100.00 & 6.39 \\
\hline 80 & 12 & 40 & & & 125.00 & 539.98 & 125.00 & 7.99 \\
\hline 80 & 12 & 40 & & & 150.00 & 539.98 & 150.00 & 9.58 \\
\hline 80 & 12 & 40 & & & 175.00 & 539.98 & 175.00 & 11.18 \\
\hline 90 & 12 & 40 & \multirow{4}{*}{0.65} & \multirow{4}{*}{0.38} & 100.00 & 584.09 & 100.00 & 6.39 \\
\hline 90 & 12 & 40 & & & 125.00 & 584.09 & 125.00 & 7.99 \\
\hline 90 & 12 & 40 & & & 150.00 & 584.09 & 150.00 & 9.58 \\
\hline 90 & 12 & 40 & & & 175.00 & 584.09 & 175.00 & 11.18 \\
\hline
\end{tabular}

Table 7

Results of MC 2010 for LoA III

\begin{tabular}{|c|c|c|c|c|c|c|c|c|c|c|c|c|}
\hline $\begin{array}{c}f_{c k} \\
(M P a)\end{array}$ & $\begin{array}{c}b_{w} \\
(\mathrm{~cm})\end{array}$ & $\begin{array}{c}d \\
(\mathrm{~cm})\end{array}$ & $\theta_{\text {min }}$ & $\varepsilon_{\mathrm{x}}$ & $\mathbf{k}_{\varepsilon}$ & $\mathrm{k}_{\mathrm{c}}$ & $\mathrm{k}_{\mathrm{v}}$ & $\begin{array}{c}V_{\mathrm{Ed}} \\
(\mathrm{kN})\end{array}$ & $\begin{array}{l}\mathrm{V}_{\mathrm{Rd}, \text { min }} \\
(\mathrm{kN})\end{array}$ & $\begin{array}{l}V_{\mathrm{Rd}, \mathrm{c}} \\
(\mathrm{kN})\end{array}$ & $\begin{array}{l}\mathrm{V}_{\mathrm{Rd}, \mathrm{s}} \\
(\mathrm{kN})\end{array}$ & $\begin{array}{c}A_{\mathrm{sw}} \\
\left(\mathrm{cm}^{2} / \mathrm{m}\right)\end{array}$ \\
\hline 55 & 12 & 40 & 30 & \multirow{4}{*}{0.001} & \multirow{4}{*}{0.65} & \multirow{4}{*}{0.53} & 0.116 & 100.00 & 364.27 & 24.79 & 75.21 & 4.80 \\
\hline 55 & 12 & 40 & 30 & & & & 0.105 & 125.00 & 364.27 & 22.45 & 102.55 & 6.55 \\
\hline 55 & 12 & 40 & 30 & & & & 0.094 & 150.00 & 364.27 & 20.10 & 129.90 & 8.30 \\
\hline 55 & 12 & 40 & 30 & & & & 0.083 & 175.00 & 364.27 & 17.76 & 157.24 & 10.05 \\
\hline 60 & 12 & 40 & 30 & \multirow{4}{*}{0.001} & \multirow{4}{*}{0.65} & \multirow{4}{*}{0.52} & 0.119 & 100.00 & 386.02 & 26.45 & 73.55 & 4.70 \\
\hline 60 & 12 & 40 & 30 & & & & 0.108 & 125.00 & 386.02 & 24.14 & 100.86 & 6.44 \\
\hline 60 & 12 & 40 & 30 & & & & 0.098 & 150.00 & 386.02 & 21.82 & 128.18 & 8.19 \\
\hline 60 & 12 & 40 & 30 & & & & 0.087 & 175.00 & 386.02 & 19.51 & 155.49 & 9.93 \\
\hline 70 & 12 & 40 & 30 & \multirow{4}{*}{0.001} & \multirow{4}{*}{0.65} & \multirow{4}{*}{0.49} & 0.123 & 100.00 & 427.80 & 28.25 & 71.75 & 4.58 \\
\hline 70 & 12 & 40 & 30 & & & & 0.113 & 125.00 & 427.80 & 26.09 & 98.91 & 6.32 \\
\hline 70 & 12 & 40 & 30 & & & & 0.104 & 150.00 & 427.80 & 23.94 & 126.06 & 8.05 \\
\hline 70 & 12 & 40 & 30 & & & & 0.095 & 175.00 & 427.80 & 21.78 & 153.22 & 9.79 \\
\hline 80 & 12 & 40 & 30 & \multirow{4}{*}{0.001} & \multirow{4}{*}{0.65} & \multirow{4}{*}{0.47} & 0.126 & 100.00 & 467.63 & 28.98 & 71.02 & 4.54 \\
\hline 80 & 12 & 40 & 30 & & & & 0.117 & 125.00 & 467.63 & 27.01 & 97.99 & 6.26 \\
\hline 80 & 12 & 40 & 30 & & & & 0.109 & 150.00 & 467.63 & 25.04 & 124.96 & 7.98 \\
\hline 80 & 12 & 40 & 30 & & & & 0.100 & 175.00 & 467.63 & 23.07 & 151.93 & 9.71 \\
\hline 90 & 12 & 40 & 45 & \multirow{4}{*}{0.001} & \multirow{4}{*}{0.65} & \multirow{4}{*}{0.45} & 0.128 & 100.00 & 505.83 & 29.58 & 70.42 & 4.50 \\
\hline 90 & 12 & 40 & 45 & & & & 0.120 & 125.00 & 505.83 & 27.75 & 97.25 & 6.21 \\
\hline 90 & 12 & 40 & 45 & & & & 0.113 & 150.00 & 505.83 & 25.93 & 124.07 & 7.93 \\
\hline 90 & 12 & 40 & 45 & & & & 0.105 & 175.00 & 505.83 & 24.11 & 150.89 & 9.64 \\
\hline
\end{tabular}


where:

$V_{R d}$ is the design shear resistance;

$V_{R d, c}$ is the design shear resistance attributed to the concrete;

$V_{R d, s}$ is the design shear resistance provided by shear reinforcement; $\eta_{f_{c}}=\left(\frac{30}{f_{c k}}\right)^{1 / 3} \leq 1,0$.

$V_{E d}$ is the design shear force.

c) Maximum shear resistance for stirrups at $90^{\circ}$ :

$V_{R d, \max }=k_{c} \cdot \frac{f_{c k}}{\gamma_{c}} \cdot b_{w} \cdot z \cdot \operatorname{sen} \theta \cdot \cos \theta$

d) Design shear resistance provided by stirrups at $90^{\circ}$ :

$V_{R d, s}=\frac{A_{s w}}{s_{w}} \cdot f_{y w d} \cdot z \cdot \operatorname{cotg} \theta$

where:

$\mathrm{k}_{\mathrm{c}}=\mathrm{k}_{\varepsilon} \cdot \eta_{\mathrm{fc}}$ is the strength reduction factor;

e) Design shear resistance attributed to the concrete:

$V_{R d, c}=k_{v} \cdot \frac{\sqrt{f_{c k}}}{\gamma_{c}} \cdot b_{w} \cdot z$

Table 8

Results of NP EN 1992-1-1, with v

\begin{tabular}{|c|c|c|c|c|c|c|c|c|}
\hline $\begin{array}{c}f_{c k} \\
(M P a)\end{array}$ & $\begin{array}{c}f_{\text {ct,m }} \\
(\mathrm{MPa})\end{array}$ & v & $\begin{array}{c}b_{w} \\
(\mathrm{~cm})\end{array}$ & $\begin{array}{c}d \\
(\mathrm{~cm})\end{array}$ & $\begin{array}{c}V_{\mathrm{Ed}} \\
(\mathrm{kN})\end{array}$ & $\begin{array}{l}\mathrm{V}_{\mathrm{Rd}, \max } \\
(\mathrm{kN})\end{array}$ & $\begin{array}{l}V_{\text {Rd,s }} \\
(\mathrm{kN})\end{array}$ & $\begin{array}{c}A_{\mathrm{sw}} \\
\left(\mathrm{cm}^{2} / \mathrm{m}\right)\end{array}$ \\
\hline 55 & 4.21 & 0.468 & 12 & 40 & 100.00 & 370.66 & 100.00 & 6.39 \\
\hline 55 & 4.21 & 0.468 & 12 & 40 & 125.00 & 370.66 & 125.00 & 7.99 \\
\hline 55 & 4.21 & 0.468 & 12 & 40 & 150.00 & 370.66 & 150.00 & 9.58 \\
\hline 55 & 4.21 & 0.468 & 12 & 40 & 175.00 & 370.66 & 175.00 & 11.18 \\
\hline 60 & 4.35 & 0.456 & 12 & 40 & 100.00 & 393.98 & 100.00 & 6.39 \\
\hline 60 & 4.35 & 0.456 & 12 & 40 & 125.00 & 393.98 & 125.00 & 7.99 \\
\hline 60 & 4.35 & 0.456 & 12 & 40 & 150.00 & 393.98 & 150.00 & 9.58 \\
\hline 60 & 4.35 & 0.456 & 12 & 40 & 175.00 & 393.98 & 175.00 & 11.18 \\
\hline 70 & 4.61 & 0.432 & 12 & 40 & 100.00 & 435.46 & 100.00 & 6.39 \\
\hline 70 & 4.61 & 0.432 & 12 & 40 & 125.00 & 435.46 & 125.00 & 7.99 \\
\hline 70 & 4.61 & 0.432 & 12 & 40 & 150.00 & 435.46 & 150.00 & 9.58 \\
\hline 70 & 4.61 & 0.432 & 12 & 40 & 175.00 & 435.46 & 175.00 & 11.18 \\
\hline 80 & 4.84 & 0.408 & 12 & 40 & 100.00 & 470.02 & 100.00 & 6.39 \\
\hline 80 & 4.84 & 0.408 & 12 & 40 & 125.00 & 470.02 & 125.00 & 7.99 \\
\hline 80 & 4.84 & 0.408 & 12 & 40 & 150.00 & 470.02 & 150.00 & 9.58 \\
\hline 80 & 4.84 & 0.408 & 12 & 40 & 175.00 & 470.02 & 175.00 & 11.18 \\
\hline 90 & 5.04 & 0.384 & 12 & 40 & 100.00 & 497.66 & 100.00 & 6.39 \\
\hline 90 & 5.04 & 0.384 & 12 & 40 & 125.00 & 497.66 & 125.00 & 7.99 \\
\hline 90 & 5.04 & 0.384 & 12 & 40 & 150.00 & 497.66 & 150.00 & 9.58 \\
\hline 90 & 5.04 & 0.384 & 12 & 40 & 175.00 & 497.66 & 175.00 & 11.18 \\
\hline
\end{tabular}

Table 9

Results of NP EN 1992-1-1, with v

\begin{tabular}{|c|c|c|c|c|c|c|c|}
\hline $\begin{array}{c}f_{c k} \\
(M P a)\end{array}$ & $v_{1}$ & $\begin{array}{c}b_{w} \\
(\mathrm{~cm})\end{array}$ & $\begin{array}{c}d \\
(\mathrm{~cm})\end{array}$ & $\begin{array}{l}\mathrm{V}_{\mathrm{Ed}} \\
(\mathrm{kN})\end{array}$ & $\begin{array}{l}\mathrm{V}_{\mathrm{Rd}, \max } \\
(\mathrm{kN})\end{array}$ & $\begin{array}{l}\mathrm{V}_{\mathrm{Rd}, \mathrm{s}} \\
(\mathrm{kN})\end{array}$ & $\begin{array}{c}A_{\mathrm{sw}} \\
\left(\mathrm{cm}^{2} / \mathrm{m}\right)\end{array}$ \\
\hline 55 & 0.60 & 12 & 40 & 100.00 & 475.20 & 100.00 & 6.94 \\
\hline 55 & 0.60 & 12 & 40 & 125.00 & 475.20 & 125.00 & 8.68 \\
\hline 55 & 0.60 & 12 & 40 & 150.00 & 475.20 & 150.00 & 10.42 \\
\hline 55 & 0.60 & 12 & 40 & 175.00 & 475.20 & 175.00 & 12.15 \\
\hline 60 & 0.60 & 12 & 40 & 100.00 & 518.40 & 100.00 & 6.94 \\
\hline 60 & 0.60 & 12 & 40 & 150.00 & 518.40 & 150.00 & 10.42 \\
\hline 60 & 0.60 & 12 & 40 & 175.00 & 518.40 & 175.00 & 12.15 \\
\hline 70 & 0.55 & 12 & 40 & 100.00 & 554.40 & 100.00 & 6.94 \\
\hline 70 & 0.55 & 12 & 40 & 125.00 & 554.40 & 125.00 & 8.68 \\
\hline 70 & 0.55 & 12 & 40 & 150.00 & 554.40 & 150.00 & 10.42 \\
\hline 70 & 0.55 & 12 & 40 & 175.00 & 554.40 & 175.00 & 12.15 \\
\hline 80 & 0.50 & 12 & 40 & 100.00 & 576.00 & 100.00 & 6.94 \\
\hline 80 & 0.50 & 12 & 40 & 150.00 & 576.00 & 150.00 & 10.42 \\
\hline 80 & 0.50 & 12 & 40 & 175.00 & 576.00 & 175.00 & 12.15 \\
\hline 90 & 0.50 & 12 & 40 & 100.00 & 648.00 & 100.00 & 6.94 \\
\hline 90 & 0.50 & 12 & 40 & 125.00 & 648.00 & 125.00 & 8.68 \\
\hline 90 & 0.50 & 12 & 40 & 150.00 & 648.00 & 150.00 & 10.42 \\
\hline 90 & 0.50 & 12 & 40 & 175.00 & 648.00 & 175.00 & 12.15 \\
\hline
\end{tabular}


where:

$\sqrt{f_{c k}} \leq 8 \mathrm{MPa}$

f) Compressive stress field inclination:

$\theta_{\min } \leq \theta \leq 45^{\circ}$

The code also presents the levels-of-approximation approach. According to Muttoni and Ruiz [8], this approach is based on the use of theories based on physical parameters where the hypotheses for their applications can be refined ac- cording to the demand for accuracy. As Barros [9] highlights, the increase in the approximation level (I to IV) is followed by the increase of precision and of the time expended for the analyses.

\subsubsection{Level I Approximation}

In this level, the $V_{R d, c}$ of Equation 12 is not considered. For reinforced concrete members, $\theta_{\min }=30^{\circ}$. In addition, to calculate $V_{R d, \max }, k_{\varepsilon}=0,55$.

\section{Table 10}

Results of DIN 1045-1

\begin{tabular}{|c|c|c|c|c|c|c|c|}
\hline $\begin{array}{c}f_{c k} \\
(M P a)\end{array}$ & $\begin{array}{c}f_{\text {ct,m }} \\
(\mathrm{MPa})\end{array}$ & $\begin{array}{c}b_{w} \\
(\mathrm{~cm})\end{array}$ & $\begin{array}{c}d \\
(\mathrm{~cm})\end{array}$ & $\begin{array}{l}V_{E d} \\
(k N)\end{array}$ & $\begin{array}{l}\mathrm{V}_{\mathrm{Rd}, \max } \\
(\mathrm{kN})\end{array}$ & $\begin{array}{l}\mathrm{V}_{\mathrm{Rd}, \mathrm{s}} \\
(\mathrm{kN})\end{array}$ & $\begin{array}{c}A_{\mathrm{sw}} \\
\left(\mathrm{cm}^{2} / \mathrm{m}\right)\end{array}$ \\
\hline 55 & 4.21 & 12 & 40 & 100.00 & 891.00 & 100.00 & 6.39 \\
\hline 55 & 4.21 & 12 & 40 & 125.00 & 891.00 & 125.00 & 7.99 \\
\hline 55 & 4.21 & 12 & 40 & 150.00 & 891.00 & 150.00 & 9.58 \\
\hline 55 & 4.21 & 12 & 40 & 175.00 & 891.00 & 175.00 & 11.18 \\
\hline 60 & 4.35 & 12 & 40 & 100.00 & 972.00 & 100.00 & 6.39 \\
\hline 60 & 4.35 & 12 & 40 & 125.00 & 972.00 & 125.00 & 7.99 \\
\hline 60 & 4.35 & 12 & 40 & 150.00 & 972.00 & 150.00 & 9.58 \\
\hline 60 & 4.35 & 12 & 40 & 175.00 & 972.00 & 175.00 & 11.18 \\
\hline 70 & 4.61 & 12 & 40 & 100.00 & 1134.00 & 100.00 & 6.39 \\
\hline 70 & 4.61 & 12 & 40 & 125.00 & 1134.00 & 125.00 & 7.99 \\
\hline 70 & 4.61 & 12 & 40 & 150.00 & 1134.00 & 150.00 & 9.58 \\
\hline 70 & 4.61 & 12 & 40 & 175.00 & 1134.00 & 175.00 & 11.18 \\
\hline 80 & 4.84 & 12 & 40 & 100.00 & 1296.00 & 100.00 & 6.39 \\
\hline 80 & 4.84 & 12 & 40 & 125.00 & 1296.00 & 125.00 & 7.99 \\
\hline 80 & 4.84 & 12 & 40 & 150.00 & 1296.00 & 150.00 & 9.58 \\
\hline 80 & 4.84 & 12 & 40 & 175.00 & 1296.00 & 175.00 & 11.18 \\
\hline 90 & 5.04 & 12 & 40 & 100.00 & 1458.00 & 100.00 & 6.39 \\
\hline 90 & 5.04 & 12 & 40 & 125.00 & 1458.00 & 125.00 & 7.99 \\
\hline 90 & 5.04 & 12 & 40 & 150.00 & 1458.00 & 150.00 & 9.58 \\
\hline 90 & 5.04 & 12 & 40 & 175.00 & 1458.00 & 175.00 & 11.18 \\
\hline
\end{tabular}

Table 11

Transverse reinforcement areas $\left(\mathrm{cm}^{2} / \mathrm{m}\right)$ for beams of $20 \mathrm{~cm} \times 60 \mathrm{~cm}$

\begin{tabular}{|c|c|c|c|c|c|c|c|c|c|c|}
\hline \multirow{2}{*}{$\begin{array}{c}f_{\mathrm{ck}} \\
(\mathrm{MPa})\end{array}$} & \multirow{2}{*}{$\begin{array}{c}V_{\text {sd }} \\
(k N)\end{array}$} & \multicolumn{2}{|c|}{ NBR } & \multirow{2}{*}{ MC 1990} & \multicolumn{3}{|c|}{ MC 2010} & \multicolumn{2}{|c|}{ NP EN 1992} & \multirow{2}{*}{ DIN 1045} \\
\hline & & M I & M II & & LOA I & LOA II & LOA III & $v$ & $v_{1}$ & \\
\hline 55 & 200.00 & 3.31 & 3.31 & 8.52 & 8.52 & 8.52 & 5.68 & 8.52 & 9.26 & 8.52 \\
\hline 55 & 250.00 & 4.30 & 5.06 & 10.65 & 10.65 & 10.65 & 8.01 & 10.65 & 11.57 & 10.65 \\
\hline 55 & 300.00 & 6.43 & 7.56 & 12.78 & 12.78 & 12.78 & 10.34 & 12.78 & 13.89 & 12.78 \\
\hline 55 & 375.00 & 9.62 & 11.32 & 15.97 & 15.97 & 15.97 & 13.83 & 15.97 & 17.36 & 15.97 \\
\hline 60 & 200.00 & 3.44 & 3.44 & 8.52 & 8.52 & 8.52 & 5.51 & 8.52 & 9.26 & 8.52 \\
\hline 60 & 250.00 & 4.05 & 4.75 & 10.65 & 10.65 & 10.65 & 7.83 & 10.65 & 11.57 & 10.65 \\
\hline 60 & 300.00 & 6.18 & 7.24 & 12.78 & 12.78 & 12.78 & 10.16 & 12.78 & 13.89 & 12.78 \\
\hline 60 & 375.00 & 9.37 & 10.99 & 15.97 & 15.97 & 15.97 & 13.65 & 15.97 & 17.36 & 15.97 \\
\hline 70 & 200.00 & 3.67 & 3.67 & 8.52 & 8.52 & 8.52 & 5.33 & 8.52 & 9.26 & 8.52 \\
\hline 70 & 250.00 & 3.67 & 4.21 & 10.65 & 10.65 & 10.65 & 7.64 & 10.65 & 11.57 & 10.65 \\
\hline 70 & 300.00 & 5.74 & 6.69 & 12.78 & 12.78 & 12.78 & 9.95 & 12.78 & 13.89 & 12.78 \\
\hline 70 & 375.00 & 8.94 & 10.41 & 15.97 & 15.97 & 15.97 & 13.42 & 15.97 & 17.36 & 15.97 \\
\hline 80 & 200.00 & 3.87 & 3.87 & 8.52 & 8.52 & 8.52 & 5.26 & 8.52 & 9.26 & 8.52 \\
\hline 80 & 250.00 & 3.87 & 3.87 & 10.65 & 10.65 & 10.65 & 7.56 & 10.65 & 11.57 & 10.65 \\
\hline 80 & 300.00 & 5.36 & 6.22 & 12.78 & 12.78 & 12.78 & 9.86 & 12.78 & 13.89 & 12.78 \\
\hline 80 & 375.00 & 8.55 & 9.92 & 15.97 & 15.97 & 15.97 & 13.31 & 15.97 & 17.36 & 15.97 \\
\hline 90 & 200.00 & 4.05 & 4.05 & 8.52 & 8.52 & 8.52 & 5.21 & 8.52 & 9.26 & 8.52 \\
\hline 90 & 250.00 & 4.05 & 4.05 & 10.65 & 10.65 & 10.65 & 7.50 & 10.65 & 11.57 & 10.65 \\
\hline 90 & 300.00 & 5.01 & 5.80 & 12.78 & 12.78 & 12.78 & 9.78 & 12.78 & 13.89 & 12.78 \\
\hline 90 & 375.00 & 8.20 & 9.50 & 15.97 & 15.97 & 15.97 & 13.21 & 15.97 & 17.36 & 15.97 \\
\hline
\end{tabular}




\subsubsection{Level II Approximation}

As occurs in LoA I, the $V_{R d, c}$ portion of Equation 12 is disregarded. The minimum inclination of the compressive stress field is given by Equation 17 , but will be adopted as $30^{\circ}$. In addition, the parameter $k_{\varepsilon}$, given by Equation 18, will assume its maximum value of 0,65 on the further developed simulations.

$\theta_{\min }=20^{\circ}+10000 \varepsilon_{x}$

$k_{\varepsilon}=\frac{1}{1,2+55 \varepsilon_{1}} \leq 0,65$

\subsubsection{Level III Approximation}

In this level, the whole Equation 12 is valid. The maximum shear resistance is given by Equation 13 for $\theta=\theta_{\min }$, given by Equation 17 and admitted as $30^{\circ}$. To determinate the $V_{R d, c}$ attributed to the concrete (Equation 15), the parameter $k_{v}$ is calculated as shown on Equation 19. For the following examples, $\varepsilon_{\mathrm{x}}=0,001$.

$k_{v}=\frac{0,4}{1+1500 \varepsilon_{x}}\left(1-\frac{V_{E d}}{V_{R d, \max }\left(\theta_{\min }\right)}\right) \geq 0$

\subsubsection{Level IV approximation}

The model code does not bring specific expressions for this levelof-approximation, but establishes that the resistance of members in shear or shear combined with torsion may be determined by satisfying the applicable conditions of equilibrium and compatibility of strains and by using appropriate stress-strain models for steel and for diagonally cracked concrete.

\subsection{NP EN 1992-1-1:2010 [10]}

Ahead, the Portuguese standard considerations on shear are shown. a) Minimum shear reinforcement area: also given by Equation 11 . b) Design shear resistance:

$V_{\mathrm{Rd}}=V_{\mathrm{Rd}, \mathrm{s}}+V_{\mathrm{ccd}}+V_{\mathrm{td}}$

where:

$V_{R d, s}$ is the design value of the shear force which can be sustained by the yielding shear reinforcement;

$V_{c c d}$ is the design value of the shear component of the force in the compression area (inclined compression chord);

$V_{t d}$ is the design value of the shear component of the force in the tensile reinforcement (inclined tensile chord).

c) Concrete compression strut inclination:

$21,8^{\circ} \leq \theta \leq 45^{\circ}$

d) Design value of the maximum shear force for stirrups at $90^{\circ}$ :

$V_{\mathrm{Rd}, \max }=\propto_{\mathrm{cw}} \cdot b_{\mathrm{w}} \cdot z \cdot v_{1} \cdot \frac{f_{\mathrm{ck}}}{\gamma_{\mathrm{c}}} /(\operatorname{cotg} \theta+\operatorname{tg} \theta)$

where:

$\mathrm{v}_{1}$ is the strength reduction factor for concrete cracked in shear; $\propto_{c w}$ is the coefficient taking account of the state of the stress in the compression chord. For reinforced concrete members, $\propto_{c w}=1,0$. The value of $v_{1}$ is given by Equation 23 .

$v=0,6\left[1-\frac{f_{\mathrm{ck}}}{250}\right]$

If the design stress of the shear reinforcement is below $80 \%$ of the characteristic yield stress $f_{\mathrm{yk}}, \mathrm{v}_{1}$ may be taken as:

$v_{1}=0,6$ for $f_{c k} \leq 60 \mathrm{MPa}$

$v_{1}=0,9-f_{c k} / 200>0,5$ for $f_{c k} \geq 60 \mathrm{MPa}$

e) Shear resistance of the stirrups at $90^{\circ}$ :

$V_{\mathrm{Rd}, \mathrm{s}}=\frac{A_{\mathrm{sw}}}{s} \cdot f_{\mathrm{ywd}} \cdot z \cdot \operatorname{cotg} \theta$

f) Maximum effective cross-sectional area of the shear reinforcement for $\theta=45^{\circ}$ :

$\frac{A_{\mathrm{sw}, \max } \cdot f_{\mathrm{ywd}}}{b_{\mathrm{w}} \cdot s} \leq \frac{0,5 \cdot \propto_{\mathrm{cw}} \cdot v_{1} \cdot f_{\mathrm{cd}}}{\operatorname{sen} \alpha}$

Table 12

Transverse reinforcement areas $\left(\mathrm{cm}^{2} / \mathrm{m}\right)$ for beams of $60 \mathrm{~cm} \times 165 \mathrm{~cm}$

\begin{tabular}{|c|c|c|c|c|c|c|c|c|c|c|}
\hline \multirow{2}{*}{$\begin{array}{c}f_{c k} \\
(M P a)\end{array}$} & \multirow{2}{*}{$\begin{array}{l}V_{\mathrm{Sd}} \\
(\mathrm{kN})\end{array}$} & \multicolumn{2}{|c|}{ NBR } & \multirow{2}{*}{ MC 1990} & \multicolumn{3}{|c|}{ MC 2010} & \multicolumn{2}{|c|}{ NP EN 1992} & \multirow{2}{*}{ DIN 1045} \\
\hline & & M I & M II & & LoA I & LoA II & LoA III & $v$ & $v_{1}$ & \\
\hline 55 & 3000 & 27.41 & 32.25 & 46.46 & 46.46 & 46.46 & 39.91 & 46.46 & 50.51 & 46.46 \\
\hline 55 & 3200 & 30.50 & 35.89 & 49.56 & 49.56 & 49.56 & 43.30 & 49.56 & 53.87 & 49.56 \\
\hline 55 & 3400 & 33.60 & 39.53 & 52.66 & 52.66 & 52.66 & 46.68 & 52.66 & 57.24 & 52.66 \\
\hline 55 & 3600 & 36.69 & 43.18 & 55.76 & 55.76 & 55.76 & 50.07 & 55.76 & 60.61 & 55.76 \\
\hline 60 & 3000 & 26.67 & 31.26 & 46.46 & 46.46 & 46.46 & 39.36 & 46.46 & 50.51 & 46.46 \\
\hline 60 & 3400 & 32.86 & 38.51 & 52.66 & 52.66 & 52.66 & 46.13 & 52.66 & 57.24 & 52.66 \\
\hline 60 & 3600 & 35.96 & 42.14 & 55.76 & 55.76 & 55.76 & 49.51 & 55.76 & 60.61 & 55.76 \\
\hline 70 & 3000 & 25.36 & 29.54 & 46.46 & 46.46 & 46.46 & 38.69 & 46.46 & 50.51 & 46.46 \\
\hline 70 & 3200 & 28.45 & 33.14 & 49.56 & 49.56 & 49.56 & 42.06 & 49.56 & 53.87 & 49.56 \\
\hline 70 & 3400 & 31.55 & 36.75 & 52.66 & 52.66 & 52.66 & 45.42 & 52.66 & 57.24 & 52.66 \\
\hline 70 & 3600 & 34.64 & 40.36 & 55.76 & 55.76 & 55.76 & 48.79 & 55.76 & 60.61 & 55.76 \\
\hline 80 & 3200 & 27.29 & 31.67 & 49.56 & 49.56 & 49.56 & 41.69 & 49.56 & 53.87 & 49.56 \\
\hline 80 & 3400 & 30.39 & 35.27 & 52.66 & 52.66 & 52.66 & 45.04 & 52.66 & 57.24 & 52.66 \\
\hline 80 & 3600 & 33.48 & 38.86 & 55.76 & 55.76 & 55.76 & 48.38 & 55.76 & 60.61 & 55.76 \\
\hline 90 & 3000 & 23.16 & 26.83 & 46.46 & 46.46 & 46.46 & 38.07 & 46.46 & 50.51 & 46.46 \\
\hline 90 & 3200 & 26.25 & 30.41 & 49.56 & 49.56 & 49.56 & 41.40 & 49.56 & 53.87 & 49.56 \\
\hline 90 & 3400 & 29.35 & 34.00 & 52.66 & 52.66 & 52.66 & 44.72 & 52.66 & 57.24 & 52.66 \\
\hline 90 & 3600 & 32.45 & 37.59 & 55.76 & 55.76 & 55.76 & 48.05 & 55.76 & 60.61 & 55.76 \\
\hline
\end{tabular}




\subsection{DIN 1045-1:2001-07 [11]}

The German standard establishes the following considerations on shear design.

a) Inclination of struts:

$18,43^{\circ} \leq \theta \leq 59,88^{\circ}$

b) Design shear resistance, limited by the strength of the struts:

$V_{\mathrm{Rd}, \max }=\frac{b_{\mathrm{w}} \cdot z \cdot \alpha_{\mathrm{c}} \cdot f_{\mathrm{ck}}}{\operatorname{cotg} \theta+\operatorname{tg} \theta}$

where:

$\alpha_{c}$ is a reduction factor equal to $0.75 \eta_{1}$, that is, 0.75 for normalweight concrete.

c) Design shear resistance, limited by the capacity of the shear reinforcement:

$V_{\mathrm{Rd}, s y}=\frac{A_{\mathrm{sw}}}{s_{\mathrm{w}}} \cdot f_{\mathrm{yd}} \cdot z \cdot \operatorname{cotg} \theta$

\section{Numerical simulations of the reinforcement areas according to the design standards}

With the objective of evaluating each analyzed procedure, three situations are proposed, each one with four intensities of shear force and considering the high strength classes of concrete. For comparison purposes, the inclinations of all struts will be taken as $\theta=45^{\circ}$. The areas were calculated using the expressions presented in section 2 .

After the proposed situations, the ultimate shear strengths obtained through the codes' expressions will be evaluated.

\subsection{Exemple 01}

The first example consists of a beam of $12 \mathrm{~cm}$ width by $40 \mathrm{~cm}$, subjected to four shear force intensities: $100 \mathrm{kN}, 125 \mathrm{kN}, 150 \mathrm{kN}$ and $175 \mathrm{kN}$. The transverse reinforcement areas obtained are presented in Tables 2 to 10 .

Table 13

Ultimate shear strengths

\begin{tabular}{cccccc}
\hline \multirow{2}{*}{ Design standard } & \multicolumn{5}{c}{ V/bd (MPa) } \\
\cline { 2 - 6 } & C55 & C60 & C70 & C80 & C90 \\
\hline NBR - I & 8.27 & 8.79 & 9.72 & 10.49 & 11.11 \\
NBR - II & 8.27 & 8.79 & 9.72 & 10.49 & 11.11 \\
MC 1990 & 7.72 & 8.21 & 9.07 & 9.79 & 10.37 \\
MC 2010 LOA I & 7.41 & 7.86 & 8.71 & 9.52 & 10.30 \\
MC 2010 LOA II & 8.76 & 9.29 & 10.29 & 11.25 & 12.17 \\
MC 2010 LOA III & 7.59 & 8.04 & 8.91 & 9.74 & 10.54 \\
NP EN 1992 & 7.72 & 8.21 & 9.07 & 9.79 & 10.37 \\
NP EN 1992 & 9.90 & 10.80 & 11.55 & 12.00 & 13.50 \\
DIN 1045 & 18.56 & 20.25 & 23.63 & 27.00 & 30.38 \\
\hline
\end{tabular}

Table 14

Comparative percentages of the areas of example 01

\begin{tabular}{|c|c|c|c|c|c|c|c|c|c|c|}
\hline \multirow{3}{*}{$\begin{array}{c}f_{c k} \\
(M P a)\end{array}$} & \multirow{3}{*}{$\begin{array}{l}V_{\text {sd }} \\
(\mathrm{kN})\end{array}$} & \multicolumn{9}{|c|}{$\mathbf{A}_{\mathrm{sw}} / \mathbf{A}_{\mathrm{sw}(\mathrm{MC} 1990)} \%$} \\
\hline & & \multicolumn{2}{|c|}{ NBR } & \multirow{2}{*}{ MC 1990} & \multicolumn{3}{|c|}{ MC 2010} & \multicolumn{2}{|c|}{ NP EN 1992} & \multirow{2}{*}{ DIN 1045} \\
\hline & & M I & M II & & LOA I & LoA II & LOA III & $v$ & $v_{1}$ & \\
\hline 55 & 100 & 40.36 & 47.49 & 100.00 & 100.00 & 100.00 & 75.21 & 100.00 & 108.70 & 100.00 \\
\hline 55 & 125 & 52.28 & 61.51 & 100.00 & 100.00 & 100.00 & 82.04 & 100.00 & 108.70 & 100.00 \\
\hline 55 & 150 & 60.22 & 70.86 & 100.00 & 100.00 & 100.00 & 86.60 & 100.00 & 108.70 & 100.00 \\
\hline 55 & 175 & 65.90 & 77.54 & 100.00 & 100.00 & 100.00 & 89.85 & 100.00 & 108.70 & 100.00 \\
\hline 60 & 100 & 38.07 & 44.61 & 100.00 & 100.00 & 100.00 & 73.55 & 100.00 & 108.70 & 100.00 \\
\hline 60 & 125 & 50.44 & 59.11 & 100.00 & 100.00 & 100.00 & 80.69 & 100.00 & 108.70 & 100.00 \\
\hline 60 & 150 & 58.69 & 68.78 & 100.00 & 100.00 & 100.00 & 85.45 & 100.00 & 108.70 & 100.00 \\
\hline 60 & 175 & 64.59 & 75.69 & 100.00 & 100.00 & 100.00 & 88.85 & 100.00 & 108.70 & 100.00 \\
\hline 70 & 100 & 34.46 & 39.54 & 100.00 & 100.00 & 100.00 & 71.75 & 100.00 & 108.70 & 100.00 \\
\hline 70 & 125 & 47.14 & 54.92 & 100.00 & 100.00 & 100.00 & 79.13 & 100.00 & 108.70 & 100.00 \\
\hline 70 & 150 & 55.94 & 65.17 & 100.00 & 100.00 & 100.00 & 84.04 & 100.00 & 108.70 & 100.00 \\
\hline 70 & 175 & 62.23 & 72.49 & 100.00 & 100.00 & 100.00 & 87.55 & 100.00 & 108.70 & 100.00 \\
\hline 80 & 100 & 36.35 & 36.35 & 100.00 & 100.00 & 100.00 & 71.02 & 100.00 & 108.70 & 100.00 \\
\hline 80 & 125 & 44.24 & 51.34 & 100.00 & 100.00 & 100.00 & 78.39 & 100.00 & 108.70 & 100.00 \\
\hline 80 & 150 & 53.52 & 62.12 & 100.00 & 100.00 & 100.00 & 83.31 & 100.00 & 108.70 & 100.00 \\
\hline 80 & 175 & 60.15 & 69.81 & 100.00 & 100.00 & 100.00 & 86.82 & 100.00 & 108.70 & 100.00 \\
\hline 90 & 100 & 38.05 & 38.05 & 100.00 & 100.00 & 100.00 & 70.42 & 100.00 & 108.70 & 100.00 \\
\hline 90 & 125 & 41.64 & 48.24 & 100.00 & 100.00 & 100.00 & 77.80 & 100.00 & 108.70 & 100.00 \\
\hline 90 & 150 & 51.36 & 59.49 & 100.00 & 100.00 & 100.00 & 82.71 & 100.00 & 108.70 & 100.00 \\
\hline 90 & 175 & 58.30 & 67.54 & 100.00 & 100.00 & 100.00 & 86.22 & 100.00 & 108.70 & 100.00 \\
\hline
\end{tabular}




\subsection{Exemple 02}

The second example consists of a beam of $20 \mathrm{~cm}$ width by $60 \mathrm{~cm}$, subjected to four shear force intensities: $200 \mathrm{kN}, 250 \mathrm{kN}, 300 \mathrm{kN}$ and $375 \mathrm{kN}$. Table 11 demonstrates the area values obtained from each normative treatment.

\subsection{Exemple 03}

The third example consists of a beam of $60 \mathrm{~cm}$ width by $165 \mathrm{~cm}$, subjected to shear forces of $3000 \mathrm{kN}, 3200 \mathrm{kN}, 3400 \mathrm{kN}$ and 3600 $\mathrm{kN}$. The calculation areas obtained are presented in Table 12.

\section{Table 15}

Comparative percentages of the areas of example 02

\begin{tabular}{|c|c|c|c|c|c|c|c|c|c|c|}
\hline \multirow{3}{*}{$\begin{array}{c}f_{c k} \\
(M P a)\end{array}$} & \multirow{3}{*}{$\begin{array}{l}V_{\text {sd }} \\
(\mathrm{kN})\end{array}$} & \multicolumn{9}{|c|}{$\mathbf{A}_{\mathrm{sw}} / \mathbf{A}_{\mathrm{sw}(\mathrm{MC} 1990)} \%$} \\
\hline & & \multicolumn{2}{|c|}{ NBR } & \multirow{2}{*}{ MC 1990} & \multicolumn{3}{|c|}{ MC 2010} & \multicolumn{2}{|c|}{ NP EN 1992} & \multirow{2}{*}{ DIN 1045} \\
\hline & & M I & M II & & LoA I & LoA II & LOA III & $v$ & $v_{1}$ & \\
\hline 55 & 200 & 38.88 & 38.88 & 100.00 & 100.00 & 100.00 & 66.66 & 100.00 & 108.70 & 100.00 \\
\hline 55 & 250 & 40.36 & 47.49 & 100.00 & 100.00 & 100.00 & 75.21 & 100.00 & 108.70 & 100.00 \\
\hline 55 & 300 & 50.29 & 59.17 & 100.00 & 100.00 & 100.00 & 80.90 & 100.00 & 108.70 & 100.00 \\
\hline 55 & 375 & 60.22 & 70.86 & 100.00 & 100.00 & 100.00 & 86.60 & 100.00 & 108.70 & 100.00 \\
\hline 60 & 200 & 40.38 & 40.38 & 100.00 & 100.00 & 100.00 & 64.63 & 100.00 & 108.70 & 100.00 \\
\hline 60 & 250 & 38.07 & 44.61 & 100.00 & 100.00 & 100.00 & 73.55 & 100.00 & 108.70 & 100.00 \\
\hline 60 & 300 & 48.38 & 56.70 & 100.00 & 100.00 & 100.00 & 79.50 & 100.00 & 108.70 & 100.00 \\
\hline 60 & 375 & 58.69 & 68.78 & 100.00 & 100.00 & 100.00 & 85.45 & 100.00 & 108.70 & 100.00 \\
\hline 70 & 200 & 43.07 & 43.07 & 100.00 & 100.00 & 100.00 & 62.54 & 100.00 & 108.70 & 100.00 \\
\hline 70 & 250 & 34.46 & 39.54 & 100.00 & 100.00 & 100.00 & 71.75 & 100.00 & 108.70 & 100.00 \\
\hline 70 & 300 & 44.94 & 52.35 & 100.00 & 100.00 & 100.00 & 77.90 & 100.00 & 108.70 & 100.00 \\
\hline 70 & 375 & 55.94 & 65.17 & 100.00 & 100.00 & 100.00 & 84.04 & 100.00 & 108.70 & 100.00 \\
\hline 80 & 200 & 45.44 & 45.44 & 100.00 & 100.00 & 100.00 & 61.80 & 100.00 & 108.70 & 100.00 \\
\hline 80 & 250 & 36.35 & 36.35 & 100.00 & 100.00 & 100.00 & 71.02 & 100.00 & 108.70 & 100.00 \\
\hline 80 & 300 & 41.92 & 48.65 & 100.00 & 100.00 & 100.00 & 77.16 & 100.00 & 108.70 & 100.00 \\
\hline 80 & 375 & 53.52 & 62.12 & 100.00 & 100.00 & 100.00 & 83.31 & 100.00 & 108.70 & 100.00 \\
\hline 90 & 200 & 47.56 & 47.56 & 100.00 & 100.00 & 100.00 & 61.21 & 100.00 & 108.70 & 100.00 \\
\hline 90 & 250 & 38.05 & 38.05 & 100.00 & 100.00 & 100.00 & 70.42 & 100.00 & 108.70 & 100.00 \\
\hline 90 & 300 & 39.21 & 45.42 & 100.00 & 100.00 & 100.00 & 76.57 & 100.00 & 108.70 & 100.00 \\
\hline 90 & 375 & 51.36 & 59.49 & 100.00 & 100.00 & 100.00 & 82.71 & 100.00 & 108.70 & 100.00 \\
\hline
\end{tabular}

\section{Table 16}

Comparative percentages of the areas of example 03

\begin{tabular}{|c|c|c|c|c|c|c|c|c|c|c|}
\hline \multirow{3}{*}{$\begin{array}{c}f_{c k} \\
(M P a)\end{array}$} & \multirow{3}{*}{$\begin{array}{l}V_{\text {sd }} \\
(\mathrm{kN})\end{array}$} & \multicolumn{9}{|c|}{$\mathbf{A}_{\mathrm{sw}} / \mathbf{A}_{\mathrm{sw}(\mathrm{MC} 1990)} \%$} \\
\hline & & \multicolumn{2}{|c|}{ NBR } & \multirow{2}{*}{ MC 1990} & \multicolumn{3}{|c|}{ MC 2010} & \multicolumn{2}{|c|}{ NP EN 1992} & \multirow{2}{*}{ DIN 1045} \\
\hline & & M I & M II & & LOA I & LoA II & LOA III & $v$ & $v_{1}$ & \\
\hline 55 & 3000 & 58.98 & 69.40 & 100.00 & 100.00 & 100.00 & 85.89 & 100.00 & 108.70 & 100.00 \\
\hline 55 & 3200 & 61.54 & 72.41 & 100.00 & 100.00 & 100.00 & 87.36 & 100.00 & 108.70 & 100.00 \\
\hline 55 & 3400 & 63.80 & 75.07 & 100.00 & 100.00 & 100.00 & 88.65 & 100.00 & 108.70 & 100.00 \\
\hline 55 & 3600 & 65.81 & 77.43 & 100.00 & 100.00 & 100.00 & 89.80 & 100.00 & 108.70 & 100.00 \\
\hline 60 & 3000 & 57.40 & 67.27 & 100.00 & 100.00 & 100.00 & 84.71 & 100.00 & 108.70 & 100.00 \\
\hline 60 & 3200 & 60.06 & 70.39 & 100.00 & 100.00 & 100.00 & 86.24 & 100.00 & 108.70 & 100.00 \\
\hline 60 & 3400 & 62.41 & 73.14 & 100.00 & 100.00 & 100.00 & 87.59 & 100.00 & 108.70 & 100.00 \\
\hline 60 & 3600 & 64.50 & 75.58 & 100.00 & 100.00 & 100.00 & 88.80 & 100.00 & 108.70 & 100.00 \\
\hline 70 & 3000 & 54.57 & 63.57 & 100.00 & 100.00 & 100.00 & 83.27 & 100.00 & 108.70 & 100.00 \\
\hline 70 & 3200 & 57.41 & 66.87 & 100.00 & 100.00 & 100.00 & 84.86 & 100.00 & 108.70 & 100.00 \\
\hline 70 & 3400 & 59.91 & 69.79 & 100.00 & 100.00 & 100.00 & 86.25 & 100.00 & 108.70 & 100.00 \\
\hline 70 & 3600 & 62.13 & 72.38 & 100.00 & 100.00 & 100.00 & 87.50 & 100.00 & 108.70 & 100.00 \\
\hline 80 & 3000 & 52.07 & 60.43 & 100.00 & 100.00 & 100.00 & 82.54 & 100.00 & 108.70 & 100.00 \\
\hline 80 & 3200 & 55.06 & 63.91 & 100.00 & 100.00 & 100.00 & 84.12 & 100.00 & 108.70 & 100.00 \\
\hline 80 & 3400 & 57.70 & 66.97 & 100.00 & 100.00 & 100.00 & 85.52 & 100.00 & 108.70 & 100.00 \\
\hline 80 & 3600 & 60.05 & 69.69 & 100.00 & 100.00 & 100.00 & 86.76 & 100.00 & 108.70 & 100.00 \\
\hline 90 & 3000 & 49.84 & 57.74 & 100.00 & 100.00 & 100.00 & 81.94 & 100.00 & 108.70 & 100.00 \\
\hline 90 & 3200 & 52.97 & 61.36 & 100.00 & 100.00 & 100.00 & 83.53 & 100.00 & 108.70 & 100.00 \\
\hline 90 & 3400 & 55.74 & 64.57 & 100.00 & 100.00 & 100.00 & 84.93 & 100.00 & 108.70 & 100.00 \\
\hline 90 & 3600 & 58.19 & 67.41 & 100.00 & 100.00 & 100.00 & 86.17 & 100.00 & 108.70 & 100.00 \\
\hline
\end{tabular}


Comparative analysis among standards of the area calculation of transversal reinforcement on reinforced concrete beams of high resistance subjected by shear force

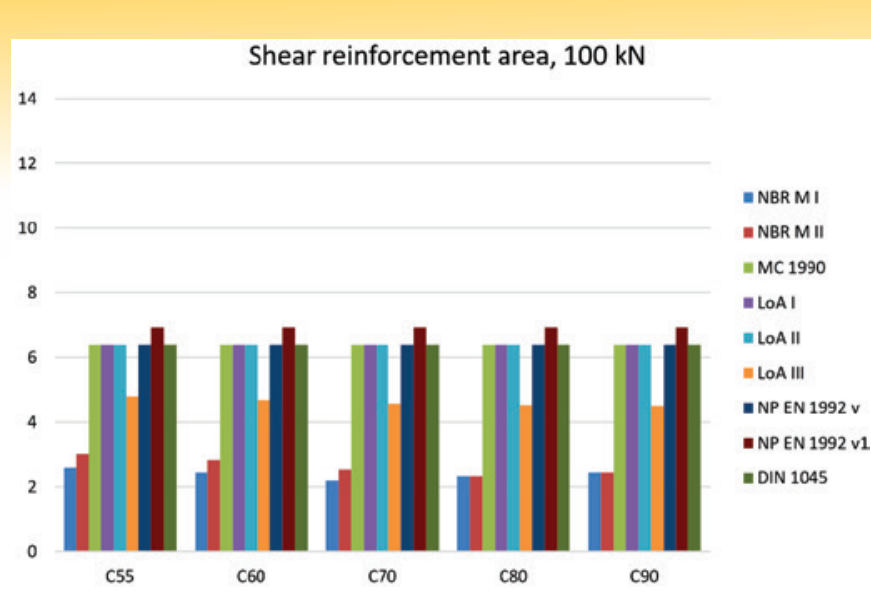

Figure 2

Comparative graph of the transverse reinforcement areas $\left(\mathrm{cm}^{2} / \mathrm{m}\right)$ of example 01 (beams of $12 \mathrm{~cm}$ by $40 \mathrm{~cm}$ ), for applied shear force of $100 \mathrm{kN}$

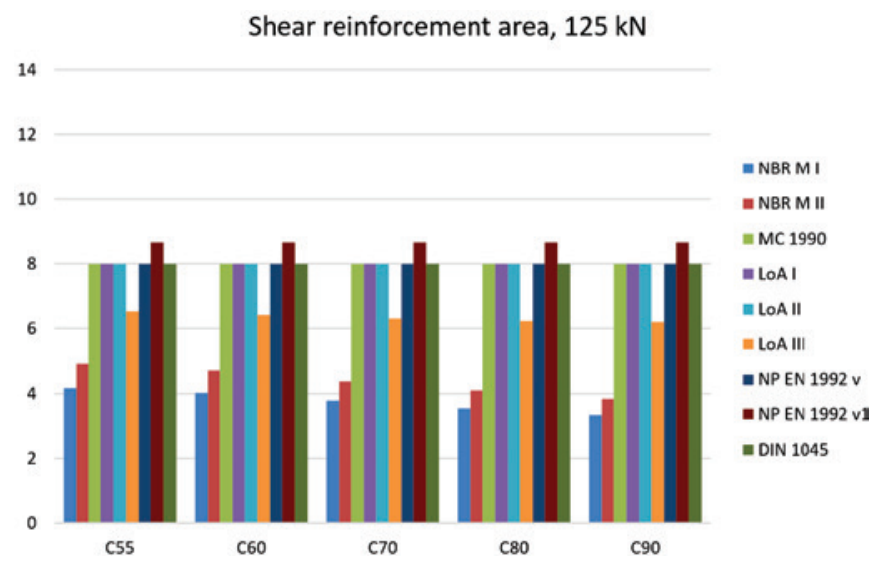

Figure 3

Comparative graph of the transverse reinforcement areas $\left(\mathrm{cm}^{2} / \mathrm{m}\right)$ of example 01 (beams of $12 \mathrm{~cm}$ by $40 \mathrm{~cm}$ ), for applied shear force of $125 \mathrm{kN}$

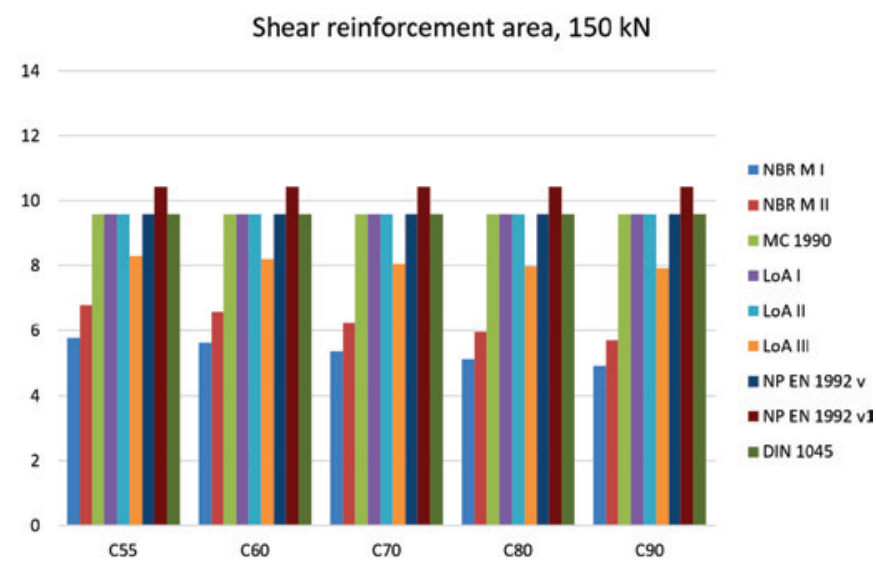

Figure 4

Comparative graph of the transverse reinforcement areas $\left(\mathrm{cm}^{2} / \mathrm{m}\right)$ of example 01 (beams of $12 \mathrm{~cm}$ by $40 \mathrm{~cm}$ ), for applied shear force of $150 \mathrm{kN}$

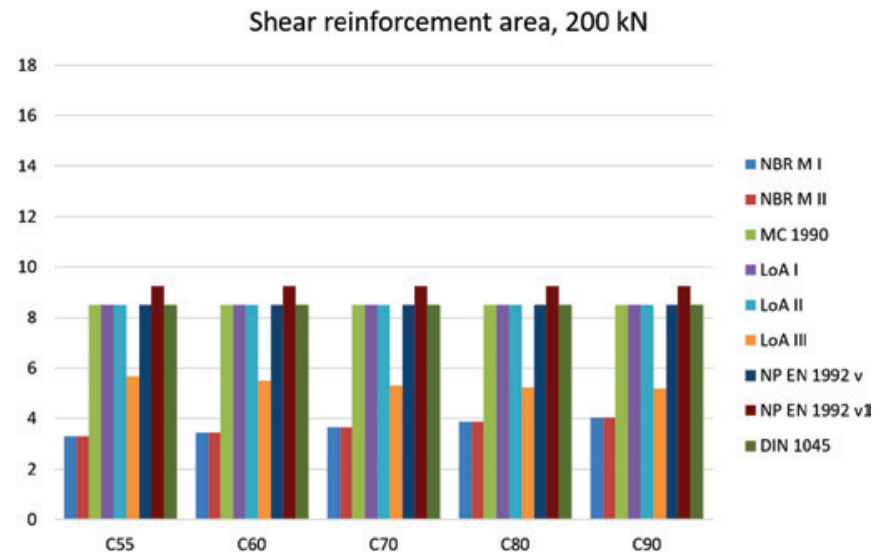

Figure 6

Comparative graph of the transverse reinforcement areas $\left(\mathrm{cm}^{2} / \mathrm{m}\right)$ of example 02 (beams of $20 \mathrm{~cm}$ by $60 \mathrm{~cm}$ ), for applied shear force of $200 \mathrm{kN}$

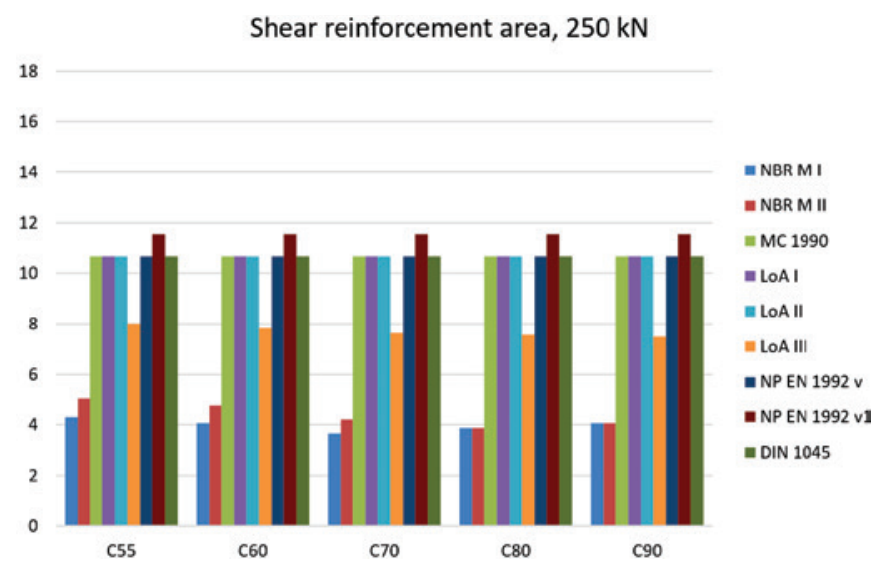

Figure 7

Comparative graph of the transverse reinforcement areas $\left(\mathrm{cm}^{2} / \mathrm{m}\right)$ of example 02 (beams of $20 \mathrm{~cm}$ by $60 \mathrm{~cm}$ ), for applied shear force of $250 \mathrm{kN}$ 


\subsection{Ultimate shear strength}

Table 13 shows the ultimate shear strengths, as stresses (MPa), for each concrete class, obtained through the studied normative procedures' expressions.

\subsection{Comparison of results}

Based on the obtained results, the comparative Tables 14, 15 and 16 , respectively related to examples 01,02 and 03 , are presented. Comparative graphs are presented in Figures 2, 3, 4, 5 (example 01); 6, 7, 8, 9 (example 02) and 10, 11, 12, 13 (example 03), for each shear force intensity and cross section situation. In the Tables, the resulting areas were considered as a percentage of the calculated areas by the Model Code 1990.

In Tables 14, 15 and 16 it can be verified that the international methodologies - apart from LOA III of MC 2010 [7] and the calculation procedure of the Portuguese code [10] which uses the parameter $v_{1}$ in the calculation (destined for situations in which the design stress of the shear reinforcement are below $80 \%$ of the characteristic yield stress) - generate the same transverse reinforcement

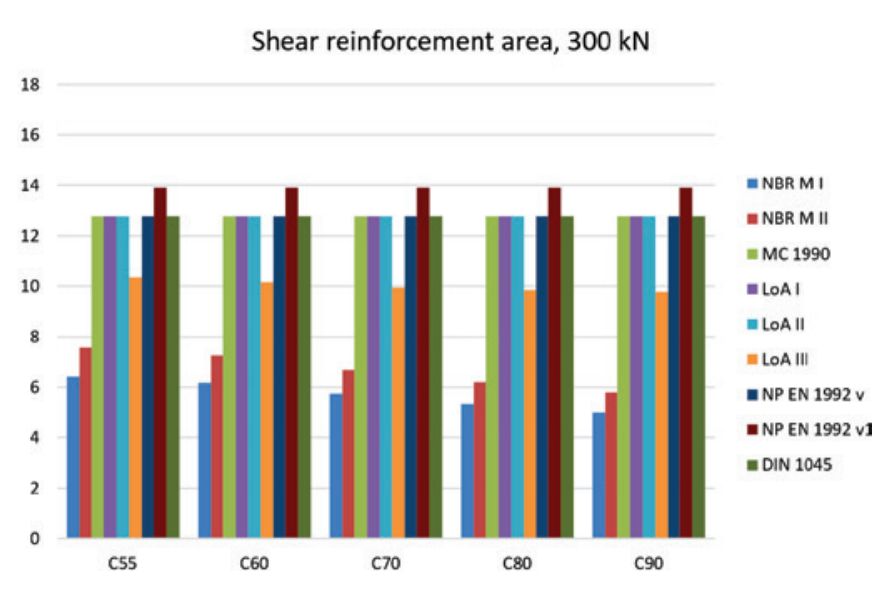

Figure 8

Comparative graph of the transverse reinforcement areas $\left(\mathrm{cm}^{2} / \mathrm{m}\right)$ of example 02 (beams of $20 \mathrm{~cm}$ by $60 \mathrm{~cm}$ ), for applied shear force of $300 \mathrm{kN}$

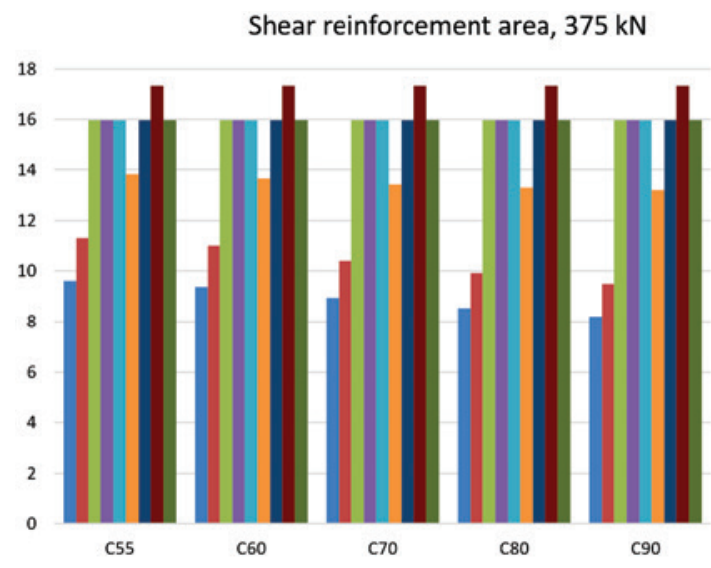

Figure 9

Comparative graph of the transverse reinforcement areas $\left(\mathrm{cm}^{2} / \mathrm{m}\right)$ of example 02 (beams of $20 \mathrm{~cm}$ by $60 \mathrm{~cm}$ ), for applied shear force of $375 \mathrm{kN}$
Shear reinforcement area, $3000 \mathrm{kN}$

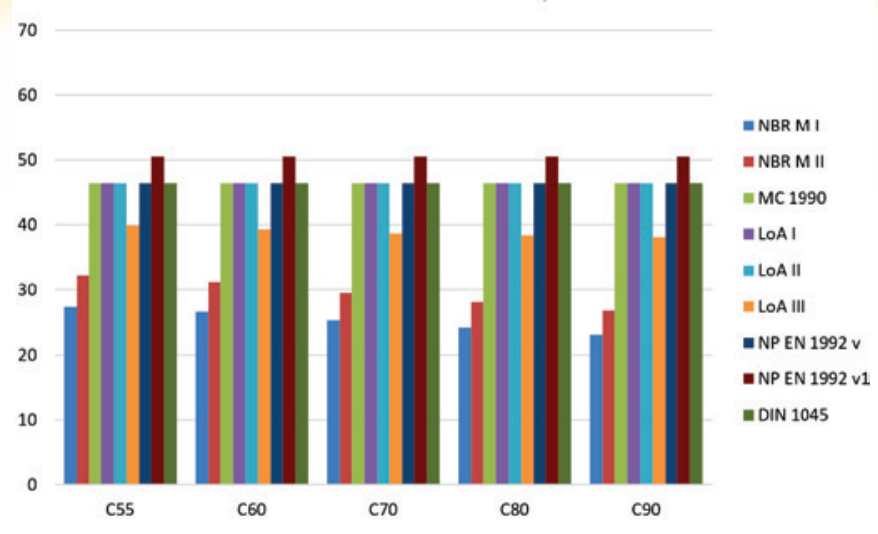

Figure 10

Comparative graph of the transverse reinforcement areas $\left(\mathrm{cm}^{2} / \mathrm{m}\right)$ of example 03 (beams of $60 \mathrm{~cm}$ por $165 \mathrm{~cm}$ ), for applied shear force of $3000 \mathrm{kN}$

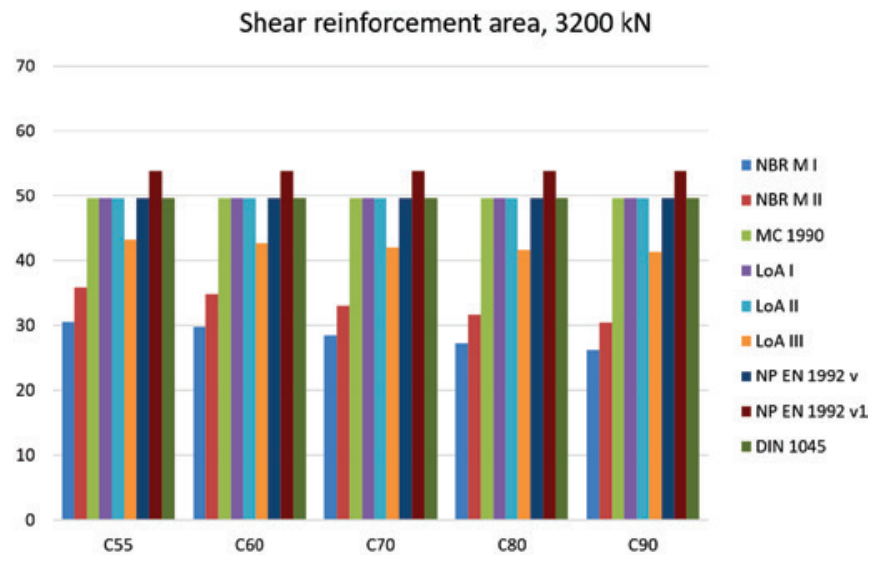

Figure 11

Comparative graph of the transverse reinforcement areas $\left(\mathrm{cm}^{2} / \mathrm{m}\right)$ of example 03 (beams of $60 \mathrm{~cm}$ por $165 \mathrm{~cm}$ ), for applied shear force of $3200 \mathrm{kN}$

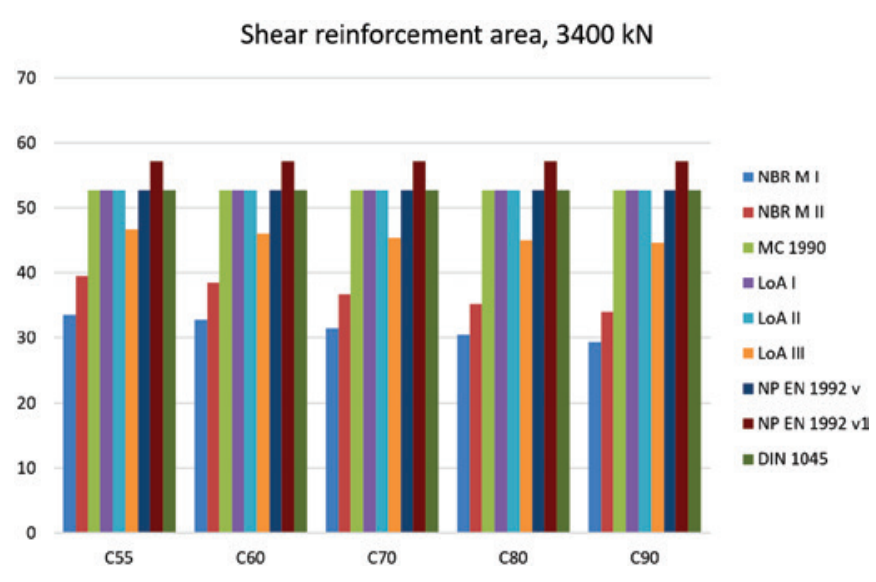

Figure 12

Comparative graph of the transverse reinforcement areas $\left(\mathrm{cm}^{2} / \mathrm{m}\right)$ of example 03 (beams of $60 \mathrm{~cm}$ por $165 \mathrm{~cm}$ ), for applied shear force of $3400 \mathrm{kN}$ 


\section{Table 17}

Results of DIN 1045-1

\begin{tabular}{|c|c|c|c|c|c|c|c|c|}
\hline \multirow{2}{*}{ Beam } & \multirow{2}{*}{$f_{c}(M P a)$} & \multirow{2}{*}{$\mathrm{b}(\mathrm{mm})$} & \multirow{2}{*}{$\mathrm{d}(\mathrm{mm})$} & \multicolumn{2}{|c|}{ Shear reinforcement } & \multicolumn{2}{|c|}{ Longitudinal reinforcement } & \multirow{2}{*}{$V_{\text {failure }}(k N)$} \\
\hline & & & & $\phi / \mathrm{s}(\mathrm{mm})$ & $\rho_{\mathrm{w}}(\mathrm{MPa})$ & $\mathbf{n} \phi$ & $\rho_{1}$ & \\
\hline $\mathrm{H} 60 / 2$ & 60.8 & 200 & 353 & $\phi 6 / 200$ & 0.747 & $2 \phi 32$ & 2.28 & 179.74 \\
\hline $\mathrm{H} 60 / 3$ & 60.8 & 200 & 351 & $\phi 8 / 210$ & 1.267 & $2 \phi 32$ & 2.29 & 258.78 \\
\hline $\mathrm{H} 60 / 4$ & 60.8 & 200 & 351 & $\phi 8 / 210$ & 1.267 & $2 \phi 32+1 \phi 25$ & 2.99 & 308.71 \\
\hline $\mathrm{H} 75 / 2$ & 68.9 & 200 & 353 & $\phi 6 / 200$ & 0.747 & $2 \phi 32$ & 2.28 & 203.94 \\
\hline $\mathrm{H} 75 / 3$ & 68.9 & 200 & 351 & $\phi 8 / 210$ & 1.267 & $2 \phi 32$ & 2.29 & 269.35 \\
\hline $\mathrm{H} 75 / 4$ & 68.9 & 200 & 351 & $\phi 8 / 210$ & 1.267 & $2 \phi 32+1 \phi 25$ & 2.99 & 255.23 \\
\hline $\mathrm{H} 100 / 2$ & 87.0 & 200 & 353 & $\phi 6 / 165$ & 0.906 & $2 \phi 32$ & 2.28 & 225.55 \\
\hline $\mathrm{H} 100 / 3$ & 87.0 & 200 & 351 & $\phi 8 / 210$ & 1.291 & $2 \phi 32$ & 2.29 & 253.64 \\
\hline $\mathrm{H} 100 / 4$ & 87.0 & 200 & 351 & $\phi 8 / 210$ & 1.291 & $2 \phi 32+1 \phi 25$ & 2.99 & 266.53 \\
\hline
\end{tabular}

areas, for the same shear intensities, beam's cross sections and inclination of the struts of $45^{\circ}$

Same as Models I and II of the NBR [5], Level III Approximation presents decreases on the required reinforcement areas for considering the concrete contribution in the design. The portions correspondent to these contributions increase while the concrete class escalates, and reduce with the rise of the applied shear forces. For all proposed situations, Models I and II of the Brazilian code generated the smallest areas.

Shear reinforcement area, $3600 \mathrm{kN}$

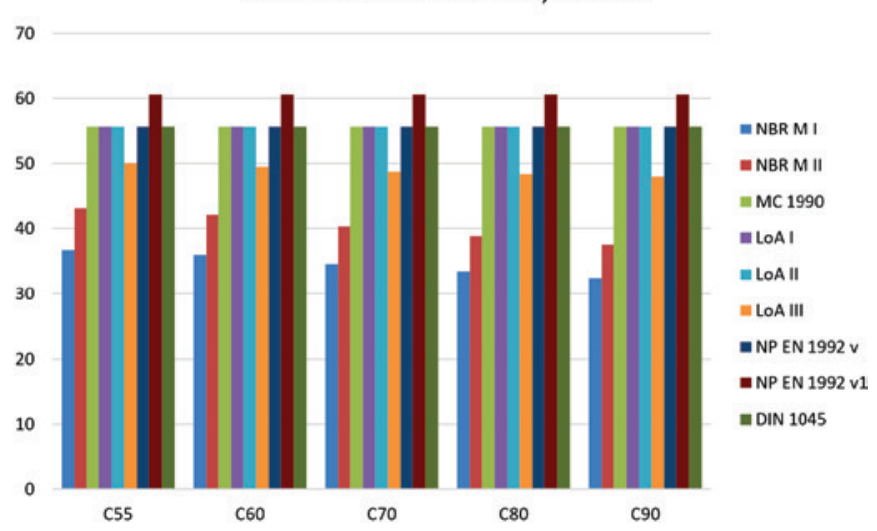

Figure 13

Comparative graph of the transverse reinforcement areas $\left(\mathrm{cm}_{2} / \mathrm{m}\right)$ of example 03 (beams of $60 \mathrm{~cm}$ por $165 \mathrm{~cm}$ ), for applied shear force of $3600 \mathrm{kN}$

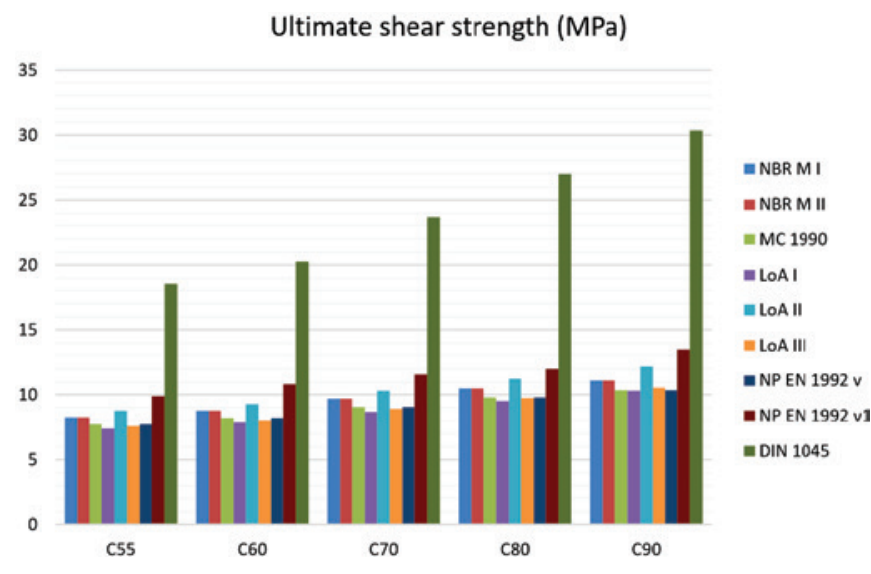

Figure 14

Comparative graph of the ultimate shear strengths $(\mathrm{MPa})$

\section{Table 18}

Properties of the transverse reinforcement bars

\begin{tabular}{cccc}
\hline Diameter - Series & Area $\left(\mathrm{mm}^{2}\right)$ & $\mathrm{f}_{\mathrm{y}}(\mathrm{MPa})$ & $\mathrm{f}_{\mathrm{u}}(\mathrm{MPa})$ \\
\hline$\phi 6-\mathrm{H} 60$ and H75 & 28.27 & 530 & 680 \\
$\phi 8-\mathrm{H} 60$ and H75 & 50.27 & 530 & 685 \\
$\phi 6-\mathrm{H} 100$ & 28.27 & 530 & 680 \\
$\phi 8-\mathrm{H} 100$ & 50.27 & 540 & 672 \\
\hline
\end{tabular}

It is possible to verify that the German standard predicts shear resistances superior to the others, as presented in Figure 14. The other codes, including the Brazilian, comprehend higher values of reduction factors over the resistance, which is significantly penalized. It must be questioned whether this higher admissible resistance of the German code is justifiable by the higher rigor demanded in executing the concrete or by other factors unrelated to the calculation procedure, which are not contemplated in the design standard.

\section{Experimental analysis}

From the previous simulations, it is observed that the NBR calculation procedure produces smaller areas than the analyzed international procedures. Among them, only LoA III of the Model Code 2010 [7] adopts the complementary mechanisms of the concrete (pin effect, aggregate gearing and arch effect) contributions. The remaining international codes presented the inconsistency of generating equal shear reinforcement areas for the same cross section and applied force, even with increase of the compression

Shear reinforcement area $\left(\mathrm{cm}^{2} / \mathrm{m}\right)$

25

20

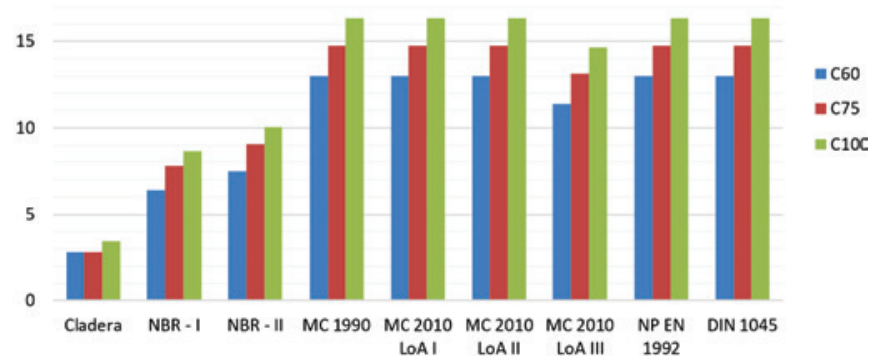

Figure 15

Comparative graph of the transverse reinforcement areas $\left(\mathrm{cm}^{2} / \mathrm{m}\right)$ of the beams of series 2 
Shear reinforcement area $\left(\mathrm{cm}^{2} / \mathrm{m}\right)$

25

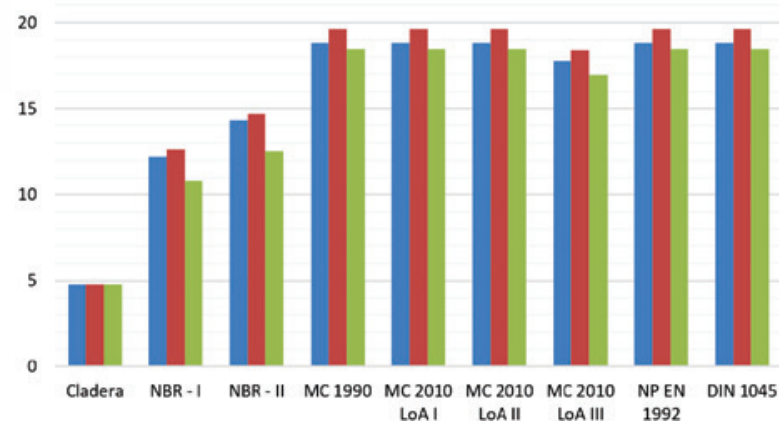

Figure 16

Comparative graph of the transverse reinforcement areas $\left(\mathrm{cm}^{2} / \mathrm{m}\right)$ of the beams of series 3

strength. Because of this, and with the intent to enrich the discussion, a comparison between the experimental results [2, 4] and the normative predictions will be performed.

Considering the results obtained by Cladera [2], the test-beams of series 2 (H60/2, H75/2 and $\mathrm{H} 100 / 2), 3$ (H60/3, H75/3 and $\mathrm{H} 100 / 3)$ and $4(\mathrm{H} 60 / 4, \mathrm{H} 75 / 4$ and $\mathrm{H} 100 / 4)$, which characteristics are ex-
Shear reinforcement area $\left(\mathrm{cm}^{2} / \mathrm{m}\right)$

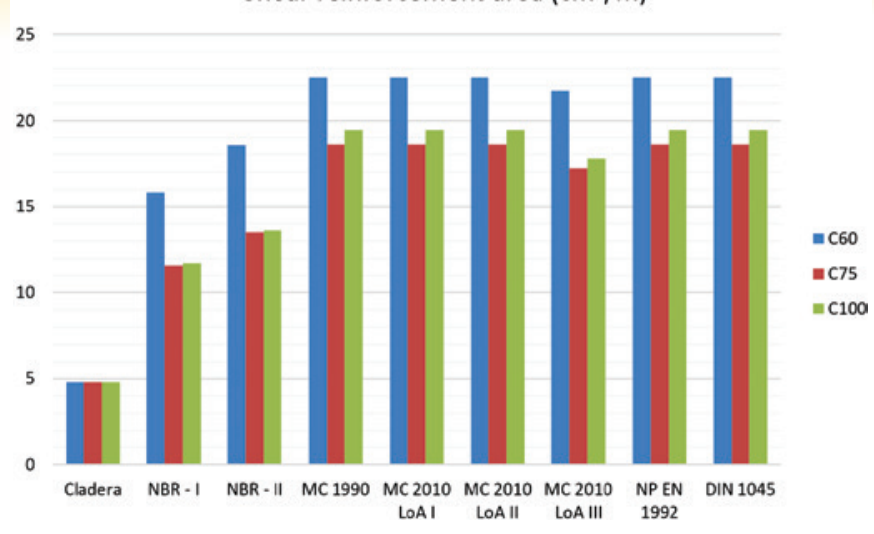

Figure 17

Comparative graph of the transverse reinforcement areas $\left(\mathrm{cm}^{2} / \mathrm{m}\right)$ of the beams of series 4

pressed in Table 17 and illustrated in Figure 1, will be contemplated. These were selected by meeting the $f_{c k}$ range of group II (between $55 \mathrm{MPa}$ and $90 \mathrm{MPa}$ ), and for being transversely reinforced, allowing the desired comparisons.

Tables 19, 20 and 21 present the areas required by the studied codes for the experimental situations [2]. These were cal-

\section{Table 19}

Transverse reinforcement areas $\left(\mathrm{cm}^{2} / \mathrm{m}\right)$ for beams of series 2

\begin{tabular}{|c|c|c|c|c|c|}
\hline Beam & $f_{c k}(M P a)$ & $b_{w}(\mathrm{~cm})$ & $\mathrm{d}(\mathrm{cm})$ & $\mathrm{V}_{\mathrm{sd}}(\mathrm{kN})$ & $A_{s w}\left(\mathrm{~cm}^{2} / \mathrm{m}\right)$ \\
\hline $\mathrm{H} 60 / 2$ & 60.8 & 20 & 35.3 & 179.74 & 2.82 \\
\hline NBR - I & 60.8 & 20 & 35.3 & 179.74 & 6.38 \\
\hline NBR - II & 60.8 & 20 & 35.3 & 179.74 & 7.47 \\
\hline MC 1990 & 60.8 & 20 & 35.3 & 179.74 & 13.01 \\
\hline MC 2010 LoA I & 60.8 & 20 & 35.3 & 179.74 & 13.01 \\
\hline MC 2010 LOA II & 60.8 & 20 & 35.3 & 179.74 & 13.01 \\
\hline MC 2010 LOA III & 60.8 & 20 & 35.3 & 179.74 & 11.38 \\
\hline NP EN 1992 & 60.8 & 20 & 35.3 & 179.74 & 13.01 \\
\hline DIN 1045 & 60.8 & 20 & 35.3 & 179.74 & 13.01 \\
\hline Beam & $f_{c k}(M P a)$ & $b_{w}(\mathrm{~cm})$ & $\mathrm{d}(\mathrm{cm})$ & $\mathrm{V}_{\mathrm{sd}}(\mathrm{kN})$ & $A_{s w}\left(\mathrm{~cm}^{2} / \mathrm{m}\right)$ \\
\hline $\mathrm{H} 75 / 2$ & 68.9 & 20 & 35.3 & 203.94 & 2.82 \\
\hline NBR - I & 68.9 & 20 & 35.3 & 203.94 & 7.77 \\
\hline NBR - II & 68.9 & 20 & 35.3 & 203.94 & 9.06 \\
\hline MC 1990 & 68.9 & 20 & 35.3 & 203.94 & 14.76 \\
\hline MC 2010 LOA I & 68.9 & 20 & 35.3 & 203.94 & 14.76 \\
\hline MC 2010 LoA II & 68.9 & 20 & 35.3 & 203.94 & 14.76 \\
\hline MC 2010 LOA III & 68.9 & 20 & 35.3 & 203.94 & 13.14 \\
\hline NP EN 1992 & 68.9 & 20 & 35.3 & 203.94 & 14.76 \\
\hline DIN 1045 & 68.9 & 20 & 35.3 & 203.94 & 14.76 \\
\hline Beam & $f_{c k}(M P a)$ & $b_{w}(\mathrm{~cm})$ & $\mathrm{d}(\mathrm{cm})$ & $\mathrm{V}_{\mathrm{sd}}(\mathrm{kN})$ & $A_{s w}\left(\mathrm{~cm}^{2} / \mathrm{m}\right)$ \\
\hline $\mathrm{H} 100 / 2$ & 87.0 & 20 & 35.3 & 225.55 & 3.42 \\
\hline NBR - I & 87.0 & 20 & 35.3 & 225.55 & 8.66 \\
\hline NBR - II & 87.0 & 20 & 35.3 & 225.55 & 10.03 \\
\hline MC 1990 & 87.0 & 20 & 35.3 & 225.55 & 16.33 \\
\hline MC 2010 LoA I & 87.0 & 20 & 35.3 & 225.55 & 16.33 \\
\hline MC 2010 LoA II & 87.0 & 20 & 35.3 & 225.55 & 16.33 \\
\hline MC 2010 LOA III & 87.0 & 20 & 35.3 & 225.55 & 14.64 \\
\hline NP EN 1992 & 87.0 & 20 & 35.3 & 225.55 & 16.33 \\
\hline DIN 1045 & 87.0 & 20 & 35.3 & 225.55 & 16.33 \\
\hline
\end{tabular}


culated considering the failure shear $\mathrm{V}_{\text {failure }}$ (Table 17), experimentally achieved, as an applied shear force and by using the effectively observed compression strength of the concrete $(60.8$ $\mathrm{MPa}, 68.9 \mathrm{MPa}$ and $87 \mathrm{MPa}$ ). It must be noted that on the standard's

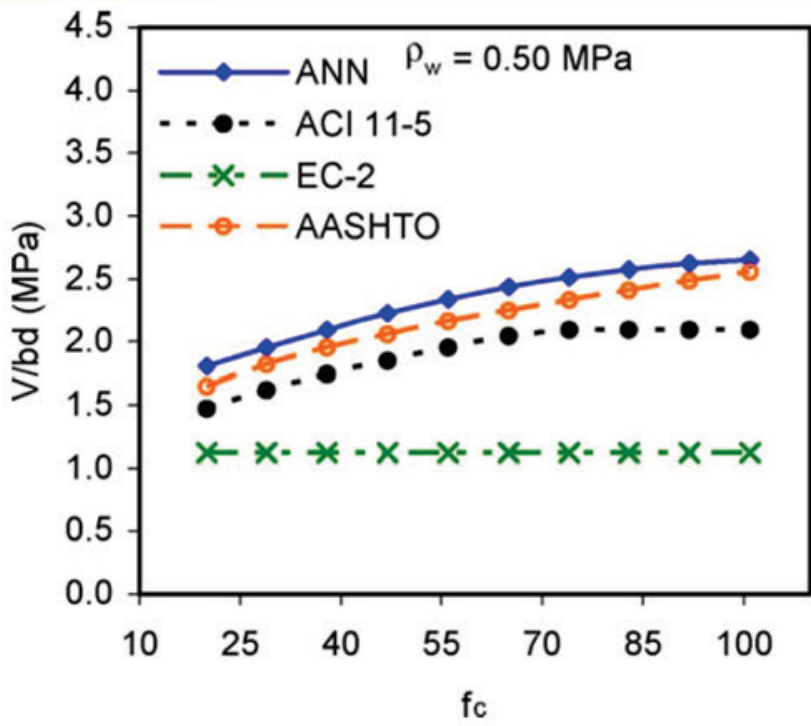

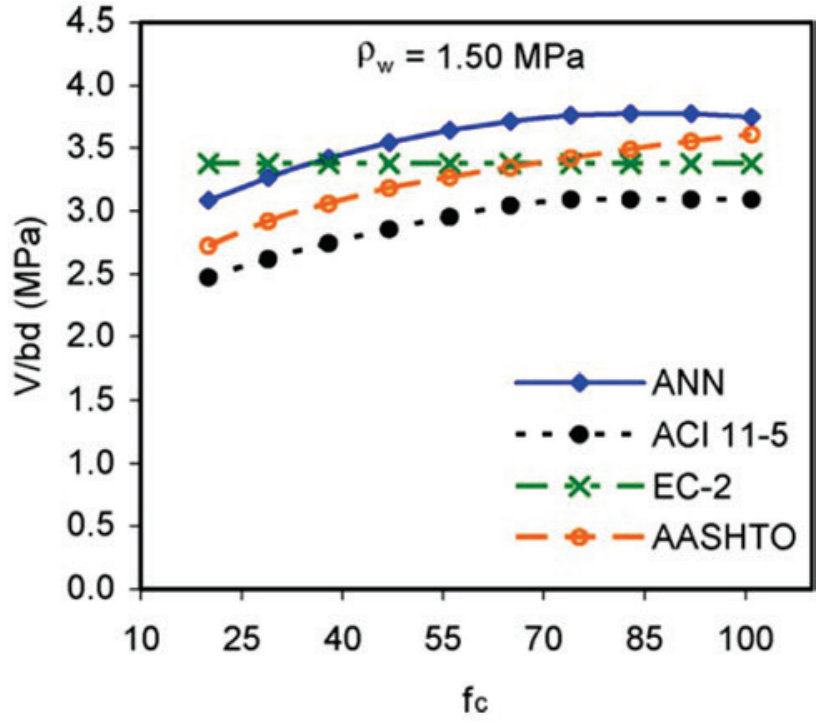

Figure 18

ANN results as compared to the ACI 11-5, EC-2 and AASHTO predictions for beams with web reinforcement. Influence of the concrete compressive strength in relation to the amount of transverse reinforcement. (Cladera \& Marí [4])

\section{Table 20}

Transverse reinforcement areas $\left(\mathrm{cm}^{2} / \mathrm{m}\right)$ for beams of series 3

\begin{tabular}{|c|c|c|c|c|c|}
\hline Beam & $f_{c k}(M P a)$ & $b_{w}(\mathrm{~cm})$ & $\mathrm{d}(\mathrm{cm})$ & $\mathrm{V}_{\mathrm{sd}}(\mathrm{kN})$ & $A_{s w}\left(\mathrm{~cm}^{2} / \mathrm{m}\right)$ \\
\hline $\mathrm{H} 60 / 3$ & 60.8 & 20 & 35.1 & 258.78 & 4.78 \\
\hline NBR - I & 60.8 & 20 & 35.1 & 258.78 & 12.20 \\
\hline NBR - ॥ & 60.8 & 20 & 35.1 & 258.78 & 14.29 \\
\hline MC 1990 & 60.8 & 20 & 35.1 & 258.78 & 18.84 \\
\hline MC 2010 LOA I & 60.8 & 20 & 35.1 & 258.78 & 18.84 \\
\hline MC 2010 LoA II & 60.8 & 20 & 35.1 & 258.78 & 18.84 \\
\hline MC 2010 LOA III & 60.8 & 20 & 35.1 & 258.78 & 17.75 \\
\hline NP EN 1992 & 60.8 & 20 & 35.1 & 258.78 & 18.84 \\
\hline DIN 1045 & 60.8 & 20 & 35.1 & 258.78 & 18.84 \\
\hline Beam & $f_{c k}(M P a)$ & $b_{w}(\mathrm{~cm})$ & $\mathrm{d}(\mathrm{cm})$ & $\mathrm{V}_{\mathrm{sd}}(\mathrm{kN})$ & $A_{s w}\left(\mathrm{~cm}^{2} / \mathrm{m}\right)$ \\
\hline $\mathrm{H} 75 / 3$ & 68.9 & 20 & 35.1 & 269.35 & 4.78 \\
\hline NBR - I & 68.9 & 20 & 35.1 & 269.35 & 12.62 \\
\hline NBR - II & 68.9 & 20 & 35.1 & 269.35 & 14.71 \\
\hline MC 1990 & 68.9 & 20 & 35.1 & 269.35 & 19.61 \\
\hline MC 2010 LOA I & 68.9 & 20 & 35.1 & 269.35 & 19.61 \\
\hline MC 2010 LoA II & 68.9 & 20 & 35.1 & 269.35 & 19.61 \\
\hline MC 2010 LOA III & 68.9 & 20 & 35.1 & 269.35 & 18.41 \\
\hline NP EN 1992 & 68.9 & 20 & 35.1 & 269.35 & 19.61 \\
\hline DIN 1045 & 68.9 & 20 & 35.1 & 269.35 & 19.61 \\
\hline Beam & $f_{c k}(M P a)$ & $b_{w}(\mathrm{~cm})$ & $\mathrm{d}(\mathrm{cm})$ & $\mathrm{V}_{\mathrm{sd}}(\mathrm{kN})$ & $A_{s w}\left(\mathrm{~cm}^{2} / \mathrm{m}\right)$ \\
\hline $\mathrm{H} 100 / 3$ & 87.0 & 20 & 35.1 & 253.64 & 4.78 \\
\hline NBR - I & 87.0 & 20 & 35.1 & 253.64 & 10.80 \\
\hline NBR - II & 87.0 & 20 & 35.1 & 253.64 & 12.51 \\
\hline MC 1990 & 87.0 & 20 & 35.1 & 253.64 & 18.47 \\
\hline MC 2010 LOA I & 87.0 & 20 & 35.1 & 253.64 & 18.47 \\
\hline MC 2010 LoA ॥ & 87.0 & 20 & 35.1 & 253.64 & 18.47 \\
\hline MC 2010 LOA III & 87.0 & 20 & 35.1 & 253.64 & 16.94 \\
\hline NP EN 1992 & 87.0 & 20 & 35.1 & 253.64 & 18.47 \\
\hline DIN 1045 & 87.0 & 20 & 35.1 & 253.64 & 18.47 \\
\hline
\end{tabular}


predictions CA-50 steel was used. On the other hand, Cladera [2] adopted the experimentally obtained yield stress, presented in Table 18, for determining the area of the transverse reinforcement. Furthermore, to make the comparison viable, like the author, no majoring factors on the applied forces or reduction coefficients on resistances were used. The areas predicted by the codes and the experimental ones correspondent to the failure shear forces are shown in Figures 15, 16 and 17. It is noticed that the predicted areas by the codes are superior to

Ultimate shear strength (MPa) - Series 2

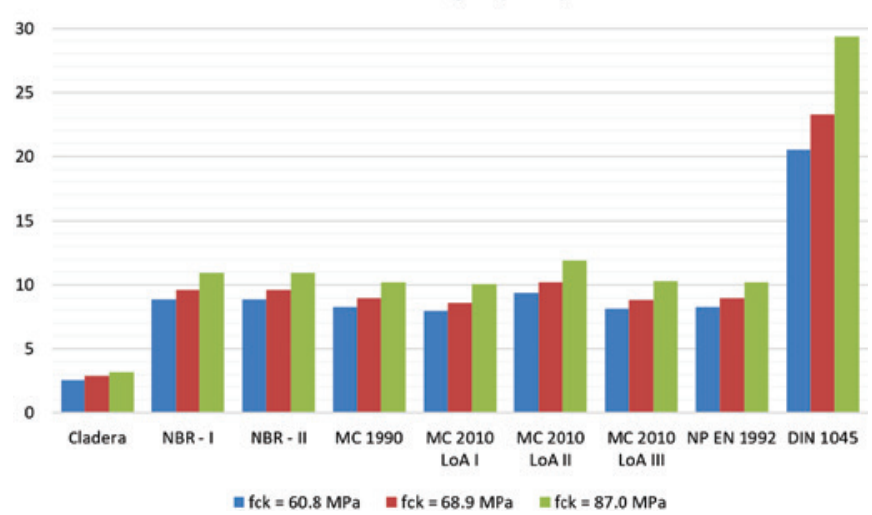

Figure 19

Comparative graph of the ultimate shear strengths $(\mathrm{MPa})$ of series 2 the experimentally required, indicating a "reserve" of resistance. According to the aforementioned by the numerical simulations of section 3 , the international standards generate greater transverse reinforcement areas then the national. The expected decrease via LOA III procedure of the Model Code 2010 [7] is highlighted, differing from the predictions of the other European codes. These last do not consider the concrete contribution, which in fact observed, as identified by Cladera \& Marí [4] (Figure 18).

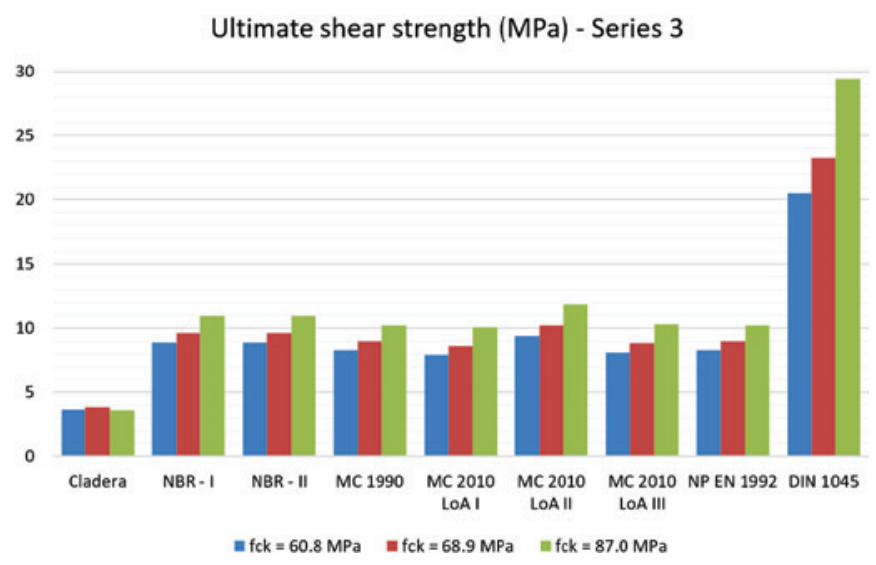

Figure 20

Comparative graph of the ultimate shear strengths $(\mathrm{MPa})$ of series 3

\section{Table 21}

Transverse reinforcement areas $\left(\mathrm{cm}^{2} / \mathrm{m}\right)$ for beams of series 4

\begin{tabular}{|c|c|c|c|c|c|}
\hline Beam & $f_{c k}(M P a)$ & $b_{w}(\mathrm{~cm})$ & $\mathrm{d}(\mathrm{cm})$ & $\mathrm{V}_{\mathrm{sd}}(\mathrm{kN})$ & $A_{s w}\left(\mathrm{~cm}^{2} / \mathrm{m}\right)$ \\
\hline $\mathrm{H} 60 / 4$ & 60.8 & 20 & 35.1 & 308.71 & 4.78 \\
\hline NBR - I & 60.8 & 20 & 35.1 & 308.71 & 15.84 \\
\hline NBR - II & 60.8 & 20 & 35.1 & 308.71 & 18.55 \\
\hline MC 1990 & 60.8 & 20 & 35.1 & 308.71 & 22.48 \\
\hline MC 2010 LOA I & 60.8 & 20 & 35.1 & 308.71 & 22.48 \\
\hline MC 2010 LoA II & 60.8 & 20 & 35.1 & 308.71 & 22.48 \\
\hline MC 2010 LOA III & 60.8 & 20 & 35.1 & 308.71 & 21.72 \\
\hline NP EN 1992 & 60.8 & 20 & 35.1 & 308.71 & 22.48 \\
\hline DIN 1045 & 60.8 & 20 & 35.1 & 308.71 & 22.48 \\
\hline Beam & $f_{c k}(M P a)$ & $b_{w}(\mathrm{~cm})$ & $\mathrm{d}(\mathrm{cm})$ & $\mathrm{V}_{\mathrm{sd}}(\mathrm{kN})$ & $A_{s w}\left(\mathrm{~cm}^{2} / \mathrm{m}\right)$ \\
\hline $\mathrm{H} 75 / 4$ & 68.9 & 20 & 35.1 & 255.23 & 4.78 \\
\hline NBR - I & 68.9 & 20 & 35.1 & 255.23 & 11.59 \\
\hline NBR - II & 68.9 & 20 & 35.1 & 255.23 & 13.51 \\
\hline MC 1990 & 68.9 & 20 & 35.1 & 255.23 & 18.58 \\
\hline MC 2010 LoA I & 68.9 & 20 & 35.1 & 255.23 & 18.58 \\
\hline MC 2010 LoA II & 68.9 & 20 & 35.1 & 255.23 & 18.58 \\
\hline MC 2010 LOA III & 68.9 & 20 & 35.1 & 255.23 & 17.29 \\
\hline NP EN 1992 & 68.9 & 20 & 35.1 & 255.23 & 18.58 \\
\hline DIN 1045 & 68.9 & 20 & 35.1 & 255.23 & 18.58 \\
\hline Beam & $f_{c k}(M P a)$ & $b_{w}(\mathrm{~cm})$ & $\mathrm{d}(\mathrm{cm})$ & $\mathrm{V}_{\mathrm{sd}}(\mathrm{kN})$ & $A_{s w}\left(\mathrm{~cm}^{2} / \mathrm{m}\right)$ \\
\hline $\mathrm{H} 100 / 4$ & 87.0 & 20 & 35.1 & 266.53 & 4.78 \\
\hline NBR - I & 87.0 & 20 & 35.1 & 266.53 & 11.73 \\
\hline NBR - II & 87.0 & 20 & 35.1 & 266.53 & 13.60 \\
\hline MC 1990 & 87.0 & 20 & 35.1 & 266.53 & 19.41 \\
\hline MC 2010 LOA I & 87.0 & 20 & 35.1 & 266.53 & 19.41 \\
\hline MC 2010 LoA II & 87.0 & 20 & 35.1 & 266.53 & 19.41 \\
\hline MC 2010 LOA III & 87.0 & 20 & 35.1 & 266.53 & 17.94 \\
\hline NP EN 1992 & 87.0 & 20 & 35.1 & 266.53 & 19.41 \\
\hline DIN 1045 & 87.0 & 20 & 35.1 & 266.53 & 19.41 \\
\hline
\end{tabular}




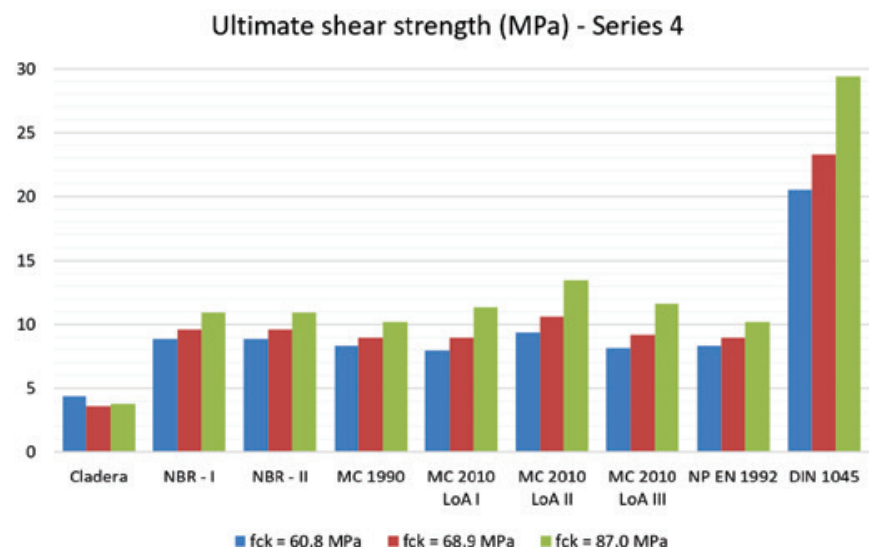

Figure 21

Comparative graph of the ultimate shear strengths (MPa) of series 4
The ANN curve - relative to the experimental results of beams of $350 \mathrm{~mm}$ of effective depth, $330 \mathrm{~mm}$ width, relation $\mathrm{a} / \mathrm{d}=3$, and longitudinal reinforcement ratio of $\rho_{1}=3 \%$ - indicates the growth of shear strength with the increase of the concrete class. Despite contemplated by the American codes ACl 318-02 and AASHTO LRDF - not studied in the present work - in a conservative manner, this behavior is not considered by the Eurocode 2 [10], which admits that variations on shear resistance are due only to the transverse reinforcement, indicated by the translation of the EC-2 curve, with the increase in the transverse reinforcement rate from $\rho_{\mathrm{w}}=0.50 \mathrm{MPa}$ to $\rho_{\mathrm{w}}=1.50 \mathrm{MPa}$.

If on one hand, most normative predictions do not consider the concrete contribution in the transverse reinforcement design, on the other, all predict ultimate shear strengths superior to those experimentally observed by Cladera [2], as presented in Table 22, which data are illustrated in Figures 19, 20 and 21. As verified in section 3.5 , the German procedure predicted the highest resistances. With the analysis of the ultimate shear strength, it is again noticeable

\section{Table 22}

Ultimate shear strengths (MPa)

\begin{tabular}{|c|c|c|c|c|}
\hline \multirow{2}{*}{ Beam } & \multirow{2}{*}{$f_{c k}(M P a)$} & \multicolumn{3}{|c|}{$\mathrm{V}_{\mathrm{Ru}}(\mathrm{MPa})$} \\
\hline & & Série 2 & Série 3 & Série 4 \\
\hline Cladera & 60.8 & 2.55 & 3.69 & 4.40 \\
\hline NBR - I & 60.8 & 8.87 & 8.87 & 8.87 \\
\hline NBR - II & 60.8 & 8.87 & 8.87 & 8.87 \\
\hline MC 1990 & 60.8 & 8.28 & 8.28 & 8.28 \\
\hline MC 2010 LOA I & 60.8 & 7.93 & 7.93 & 7.93 \\
\hline MC 2010 LoA II & 60.8 & 9.37 & 9.37 & 9.37 \\
\hline MC 2010 LoA III & 60.8 & 8.11 & 8.11 & 8.11 \\
\hline NP EN 1992 & 60.8 & 8.28 & 8.28 & 8.28 \\
\hline DIN 1045 & 60.8 & 20.52 & 20.52 & 20.52 \\
\hline \multirow{2}{*}{ Beam } & \multirow{2}{*}{$f_{c k}(M P a)$} & \multicolumn{3}{|c|}{$\mathrm{V}_{\mathrm{RU}}(\mathrm{MPa})$} \\
\hline & & Série 2 & Série 3 & Série 4 \\
\hline Cladera & 68.9 & 2.89 & 3.84 & 3.64 \\
\hline NBR - I & 68.9 & 9.63 & 9.63 & 9.63 \\
\hline NBR - II & 68.9 & 9.63 & 9.63 & 9.63 \\
\hline MC 1990 & 68.9 & 8.98 & 8.98 & 8.98 \\
\hline MC 2010 LOA I & 68.9 & 8.62 & 8.62 & 8.98 \\
\hline MC 2010 LOA II & 68.9 & 10.18 & 10.18 & 10.62 \\
\hline MC 2010 LoA III & 68.9 & 8.82 & 8.82 & 9.19 \\
\hline NP EN 1992 & 68.9 & 8.98 & 8.98 & 8.98 \\
\hline DIN 1045 & 68.9 & 23.25 & 23.25 & 23.25 \\
\hline \multirow{2}{*}{ Beam } & \multirow{2}{*}{$\mathrm{f}_{\mathrm{ck}}(\mathrm{MPa})$} & \multicolumn{3}{|c|}{$\mathrm{V}_{\mathrm{Ru}}(\mathrm{MPa})$} \\
\hline & & Série 2 & Série 3 & Série 4 \\
\hline Cladera & 87.0 & 3.19 & 3.61 & 3.80 \\
\hline NBR - I & 87.0 & 10.94 & 10.94 & 10.94 \\
\hline NBR - II & 87.0 & 10.94 & 10.94 & 10.94 \\
\hline MC 1990 & 87.0 & 10.21 & 10.21 & 10.21 \\
\hline MC 2010 LOA I & 87.0 & 10.07 & 10.07 & 11.34 \\
\hline MC 2010 LOA II & 87.0 & 11.90 & 11.90 & 13.41 \\
\hline MC 2010 LOA III & 87.0 & 10.30 & 10.30 & 11.61 \\
\hline NP EN 1992 & 87.0 & 10.21 & 10.21 & 10.21 \\
\hline DIN 1045 & 87.0 & 29.36 & 29.36 & 29.36 \\
\hline
\end{tabular}



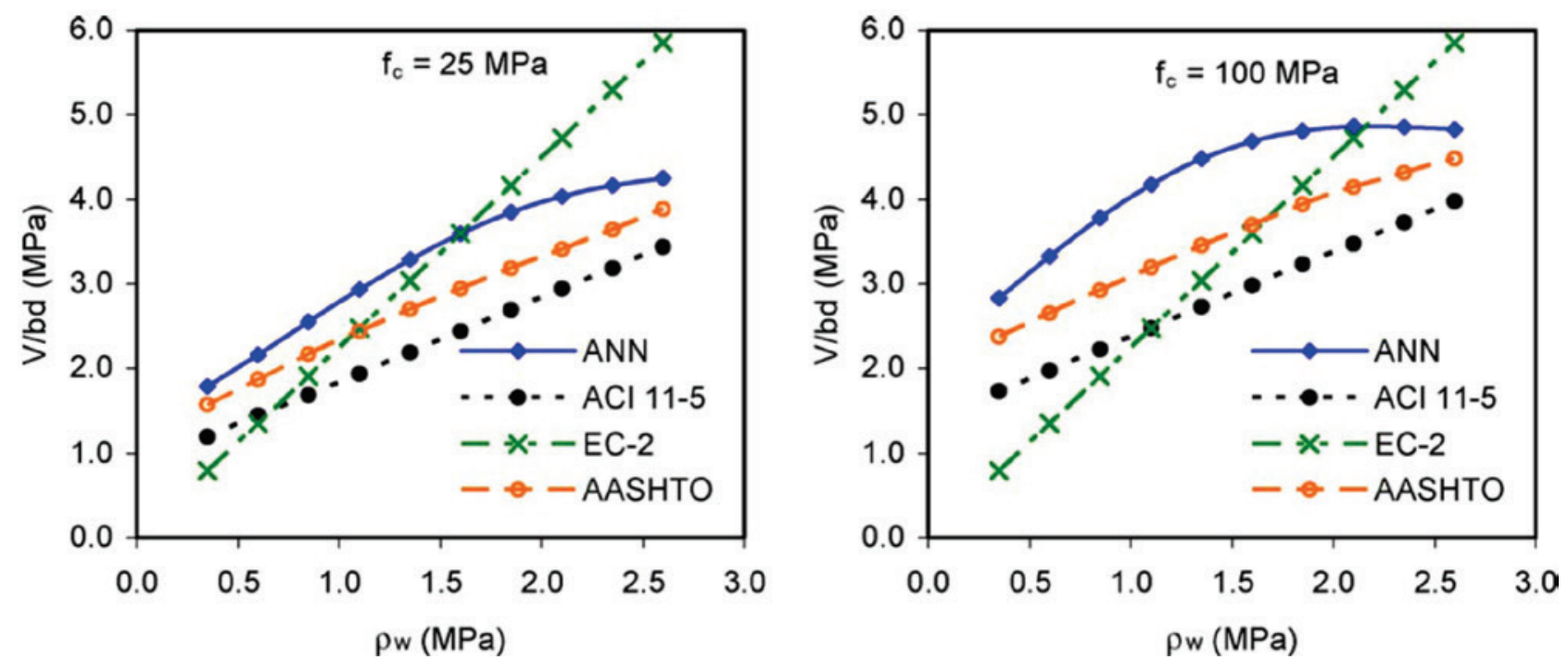

Figure 22

ANN results compared to the ACI 11-5, EC-2 and AASHTO predictions for beams with web reinforcement. Influence of the amount of shear reinforcement in relation to the concrete compressive strength (Cladera \& Marí [4])

that, despite the growth of the shear resistance with the increase of the concrete class, in accordance to the normative procedures and experimental results, this behavior is not translated into advantage in design by the European procedures (apart from LOA III).

\section{Conclusions}

Due to the diffusion of high strength concretes, it is necessary to study the normative design procedures - specifically on shear design - which encompass concretes of classes C55 to C90. This work, therefore, aimed to analytically compare the usual normative methodologies in light of experimental results [2, 4].

From the analyses, one concludes that the NBR procedure produces areas inferior to the studied international codes. Unlike the Brazilian standard [5], they do not consider (apart from LoA III) the complementary concrete mechanisms contribution (pin effect, aggregate gearing and arch effect), despite experimentally observed.

According to the data shown in Tables 14,15 and 16, one verifies that the MC 1990 [6] and MC 2010 [7] (LoA I and LOA II) calculation procedures and the Portuguese [10] (considering parameter v) and German codes [11] give the same areas for the same cross sections, shear intensities and inclination of the struts.

As verified at the $50^{\text {th }}$ Brazilian Congress on Concrete [12] for concretes of group I, the use of Model II of NBR [5] in concretes of group II for given shear force, cross section and compressed diagonal inclination of $45^{\circ}$, results in areas superior to those obtained by Model I, when these are greater than the normative minimum.

The procedures that adopt a concrete contribution present reductions in the transverse reinforcement areas with the increase of the concrete class for a same applied shear force and cross section. In general, for the same class the areas increase with the loads. Despite of not incorporating the concrete contribution in the design, the analyzed international procedures - as well as the national - predicted an increase in the ultimate shear force with the growth of the concrete class. In the performed comparisons it was detected that this same increase is superior to that experimentally obtained by Cladera [2], which reinforces the inconsistency and conservatism of these codes.

The lack of consideration of the concrete portion by part of the analyzed international codes leads to very conservative results, given that, regardless of class, for a same applied force, the areas are equal. Cladera \& Marí [4] confirm this behavior when comparing the results of the ANN with the areas predicted by the Eurocode 2, as demonstrates Figure 22.

\section{Acknowledgments}

The autors thank CAPES - Coordination for the Improvement of Higher Education Personnel, CNPq - National Council for Scientific and Technological Development and the PROPESQ of UFRN.

\section{References}

[1] SILVA, Inês Santana da. Concreto de Alta Resistência: Composição, Propriedades e Dimensionamento. 1995. $149 \mathrm{f}$. Dissertação (Mestrado) - Curso de Engenharia de Estruturas, Escola de Engenharia de São Carlos, São Carlos, 1995.

[2] CLADERA, Antoni. Shear Design of Reinforced High-Strength Concrete Beams. 2002. 159 f. Tese (Doutorado) - Curso de Enginyeria Civil, Departament D'enginyeria de La Construcció, Universitat Politècnica de Catalunya, Barcelona, 2002.

[3] ARSLAN, Güray. Shear strength of reinforced concrete beams with stirrups. Materials And Structures, [s.I.], v. 41, n. 1, p.113-122, 28 fev. 2007. Springer Nature. http://dx.doi. org/10.1617/s11527-007-9223-3. 
[4] CLADERA, A.; MARÍ, A. R.. Shear design procedure for reinforced normal and high-strength concrete beams using artificial neural networks. Part II: beams with stirrups. Engineering Structures, Elsevier, Amsterdam, v. 26, n. 7, p.927-936, 23 fev. 2004.

[5] ASSOCIAÇÃO BRASILEIRA DE NORMAS TÉCNICAS. NBR 6118: Projeto de estruturas de concreto - Procedimento. 3 ed. Rio de Janeiro, 2014.

[6] COMITE EURO-INTERNACIONAL DU BETON. MC 1990: Design Code. Lausanne, 1990.

[7] COMITE EURO-INTERNACIONAL DU BETON. MC 2010 Model Code 2010. Lausanne, 2010.

[8] MUTTONI, Aurelio; RUIZ, Miguel Fernández. The levels-ofapproximation approach in MC 2010: application to punching shear provisions. Structural Concrete,[s.I.], v. 13, n. 1, p.3241, mar. 2012. Wiley-Blackwell. http://dx.doi.org/10.1002/ suco.201100032.

[9] BARROS, Rodrigo. Como as normas brasileiras e europeias tratam o problema da força cortante em elementos lineares de concreto. TQS News, [s.i.], v. , n. 36, p.44-45, 36 mar. 2013.

[10] COMITÉ EUROPEU DE NORMALIZAÇÃO. NP EN 1992-11: Projecto de estruturas de betão. Parte 1-1: Regras gerais e regras para edifícios. Bruxelas, 2010.

[11] DEUTSCHES INSTITUT FÜR NORMUNG. DIN 10451:2001-07: Plain, reinforced and prestress concrete structures. Part 1: Design and construction. Berlin, 2001.

[12] CONGRESSO BRASILEIRO DE CONCRETO, 50., 2008, Salvador. Cálculo da área da armadura transversal em elementos lineares de concreto armado submetidas à ação de força cortante: análise comparativa entre os Modelos I e II da NBR 6118:2003. Salvador: Ibracon, 2008. 16 p. 